\title{
Survival after surgical ablation for atrial fibrillation in mitral valve surgery: Analysis from the Polish National Registry of Cardiac Surgery Procedures (KROK)
}

Piotr Suwalski, MD, PhD, ${ }^{\mathrm{a}, \mathrm{b}}$ Mariusz Kowalewski, MD, ${ }^{\mathrm{a}, \mathrm{c}}$ Marek Jasiński, MD, PhD,

Jakub Staromłyński, MD, ${ }^{a}$ Marian Zembala, MD, PhD ${ }^{\text {e,f,g }}$ Kazimierz Widenka, MD, PhD,

Mirosław Brykczyński, MD, PhD,${ }^{i}$ Jacek Skiba, MD, ${ }^{j}$ Michał Oskar Zembala, MD, PhD, e,f,g

Krzysztof Bartuś, MD, PhD, ${ }^{\mathrm{k}}$ Tomasz Hirnle, $\mathrm{MD}, \mathrm{PhD},{ }^{1}$ Inga Dziembowska, MSc, ${ }^{\mathrm{m}}$

Zdzisław Tobota, MD, ${ }^{\mathrm{n}}$ and Bohdan J. Maruszewski, $\mathrm{MD}, \mathrm{PhD},{ }^{\mathrm{n}}$ on behalf of the KROK Investigators

\section{ABSTRACT}

Objectives: Surgical ablation for atrial fibrillation $(\mathrm{AF})$ performed at the time of other valvular- or nonvalvular cardiac procedure is a mainstay of therapy; yet the data regarding its influence on remote survival are sparse. We aimed to evaluate late survival in patients undergoing mitral valve (MV) surgery with concomitant surgical ablation for AF.

Methods: Procedure-related data from the Polish National Registry of Cardiac Surgery Procedures (Krajowy Rejestr Operacji Kardiochirurgicznych) were retrospectively collected. A total of 11,381 patients with baseline AF (46.6\% men; mean age $65.6 \pm 9.0$ years) undergoing MV surgery between 2006 and 2017 in 37 reference centers across Poland and included in the registry were analyzed. Median follow-up was 5 years (mean, 4.6 years; interquartile range, 1.9-7.9 years). Cox proportional hazards models were used for computations. Propensity score matching for the comparison of MV + ablation versus MV alone was performed.

Results: Of included patients, 2449 (21.5\%) underwent surgical ablation for AF. Patients in this group were significantly younger $(63.8 \pm 8.7$ years vs $66.1 \pm 9.0$ years; $P<.001$ ) and were at lower baseline surgical risk (EuroSCORE, 2.86 vs $3.69 ; P<.001)$. During the 12 -year study period, there was a significant survival benefit (hazard ratio, $0.71 ; 95 \%$ confidence interval, $0.63-0.79 ; P<.001$ ) for MV + ablation compared with MV alone. After rigorous propensity matching (logit model, 1784 pairs) surgical ablation was associated with nearly $20 \%$ improved survival (hazard ratio, 0.82; 95\% confidence interval, 0.70-0.96; $P=.011)$. Benefit of surgical ablation was maintained in subgroup analyses, yet most benefit was appraised in low-risk patients such as those with EuroSCORE of 2 to 5 or age $<50$ years.

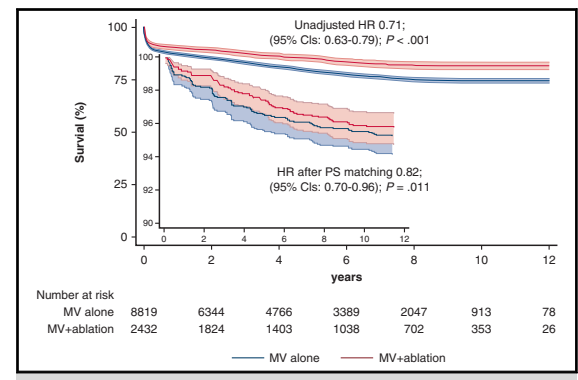

Survival analysis between mitral valve (MV) + concomitant surgical ablation versus MV surgery alone.

\section{Central Message}

In one of the largest European registries, we found that surgical ablation performed for atrial fibrillation during mitral valve surgery was associated with significantly improved longterm survival.

\section{Perspective}

Up to $40 \%$ of patients undergoing mitral valve surgery present with atrial fibrillation. Although Cox-maze III and Cox-maze IV procedures are safe and recommended in current guidelines, antiarrhythmic corrections are not always performed at index mitral valve surgery due to inconclusive data regarding long-term survival.

See Commentaries on pages 1019 and 1021.

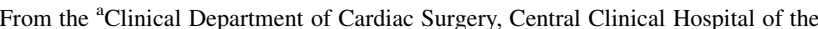
Ministry of Interior and Administration, Warsaw, Poland; ${ }^{\mathrm{b}}$ Clinical Department of Cardiac Surgery, Centre of Postgraduate Medical Education, Warsaw, Poland; ${ }^{\mathrm{c}}$ Cardiothoracic Research Centre, Collegium Medicum, Nicolaus Copernicus University, Bydgoszcz, Poland; ${ }^{\mathrm{d}}$ Department and Clinic of Cardiac Surgery, Wroclaw Medical University, Wroclaw, Poland; ${ }^{e}$ Department of Cardiac, Vascular, and Endovascular Surgery and Transplantology, School of Medicine, and ${ }^{\mathrm{f}}$ Division of Dentistry in Zabrze, Medical University of Silesia, Katowice, Poland; ${ }^{\mathrm{g}}$ Division of Cardiac Surgery, Heart and Lung Transplantation and Mechanical Circulatory

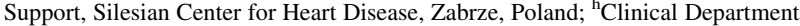
of Cardiac Surgery, District Hospital No. 2, University of Rzeszów, Rzeszów, Poland; ' Department of Cardiac Surgery, Pomeranian Medical University, Szczecin, Poland; ${ }^{j}$ Department of Cardiac Surgery, 4 Military Clinical Hospital Centre for Heart Diseases, Wroclaw, Poland; ${ }^{\mathrm{k}}$ Department of Cardiovascular Surgery and Transplantology, Jagiellonian University, John Paul II Hospital, Krakow,
}

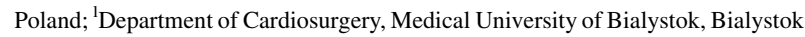
Poland; ${ }^{\mathrm{m}}$ Department of Pathophysiology, Faculty of Pharmacy, Collegium Medicum, Nicolaus Copernicus University, Torun, Poland; and ${ }^{\mathrm{n}}$ Department of Pediatric Cardiothoracic Surgery, The Children's Memorial Health Institute, Warsaw, Poland.

Read at the 98th Annual Meeting of The American Association for Thoracic Surgery, San Diego, California, April 28-May 1, 2018.

Received for publication April 26, 2018; revisions received July 3, 2018; accepted for publication July 15, 2018; available ahead of print Oct 9, 2018.

Address for reprints: Mariusz Kowalewski, MD, Clinical Department of Cardiac Surgery, Central Clinical Hospital of the Ministry of Interior and Administration, Wołoska 137 Str, 02-507 Warsaw, Poland (E-mail: kowalewskimariusz@gazeta.pl). $0022-5223 / \$ 36.00$

Copyright (c) 2018 by The American Association for Thoracic Surgery https://doi.org/10.1016/j.jtcvs.2018.07.099 


$$
\begin{aligned}
& \text { Abbreviations and Acronyms } \\
& \mathrm{AF} \quad=\text { atrial fibrillation } \\
& \text { CAD }=\text { coronary artery disease } \\
& \text { EuroSCORE }=\text { European system for cardiac } \\
& \text { operative risk evaluation } \\
& \text { HLoS = hospital length of stay } \\
& \text { IABP }=\text { intra-aortic balloon pump } \\
& \text { ICU } \quad=\text { intensive care unit } \\
& \text { KROK }=\text { Krajowy Rejestr Operacji } \\
& \text { Kardiochirurgicznych (Polish } \\
& \text { National Registry of Cardiac } \\
& \text { Surgery Procedures) } \\
& \text { LVEF = left ventricle ejection fraction } \\
& \text { MI }=\text { myocardial infarction } \\
& \text { MV } \quad=\text { mitral valve } \\
& \text { STS }=\text { Society of Thoracic Surgeons }
\end{aligned}
$$

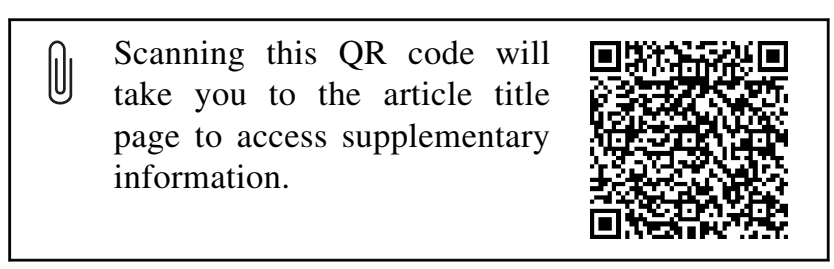

Conclusions: Concomitant surgical ablation for $\mathrm{AF}$ in patients undergoing mitral valve procedures is safe, feasible, and significantly improves late survival. (J Thorac Cardiovasc Surg 2019;157:1007-18)

Advancements in understanding of the pathogenesis of atrial fibrillation (AF) and development of new technologies have resulted in numerous improvements in the surgical ablation of AF, particularly in patients with mitral valve (MV) disease. For patients with both MV dysfunction and $\mathrm{AF}$, a variety of new approaches are available to enable a complete operation that includes both MV repair or replacement and ablation of $\mathrm{AF}$, on many occasions all done in minimally invasive fashion. ${ }^{1-4}$

Left untreated, AF increases mortality and morbidity in patients undergoing MV surgery. ${ }^{5-9}$ Although the maze procedure effectively eliminates $\mathrm{AF}$ in most of these patients, its complexity and increased operative time has precluded widespread application. ${ }^{10}$ New operations that use radiofrequency or cryoablation to create left atrial lesion, have been demonstrated to reduce long-term $\mathrm{AF}$ rates and to improve quality of life. ${ }^{11-17}$ More recently, others described that surgical ablation in patients with multiple valve disease and AF may have been associated with lower operative mortality. ${ }^{18}$ Implementation of surgical ablation is increasing, ${ }^{19}$ but up to $60 \%$ of patients have their AF left untreated. ${ }^{20}$

Performance of surgical ablation at the time of mitral disease operations recently has attained a class IA recommendation in the Society of Thoracic Surgeons (STS) $)^{21,22}$ and class IIA in the European Society of Cardiology/European Society of Cardiothoracic Surgery guidelines. ${ }^{23}$ Refinements in mapping and ablation technology are on the horizon, and these will facilitate more widespread application of minimally invasive approaches and further improve results $^{24,25}$ that to date, partially due to paucity of data, are inconclusive regarding remote safety and efficacy of surgical ablation for AF in patients undergoing MV surgery.

In the current analysis, we report long-term survival results after concomitant surgical ablation for atrial fibrillation in patients undergoing MV procedures from the Polish National Registry of Cardiac Surgery Procedures (Krajowy Rejestr Operacji Kardiochirurgicznych [KROK]).

\section{METHODS \\ Registry Design}

All data were collective in a retrospective fashion from the KROK registry (www.krok.csioz.gov.pl). The registry is an ongoing, nationwide, multi-institutional registry of heart surgery procedures in Poland. The registry is an initiative of the Club of Polish Cardiac Surgeons in cooperation with the Polish Ministry of Health that commenced in 2006 and collects data from all 37 heart surgery centers in Poland (a list of contributing centers is provided in the Acknowledgment section at the end of the article). Centers enrolling patients in the KROK registry are required to transfer the data concerning every cardiac surgery to the central database in the National Centre for Healthcare Information Systems at the Ministry of Health and are financially liable for data integrity and completeness. Follow-up data regarding mortality were obtained from the National Health Fundthe nationwide, obligatory, public health insurance institution in Poland and incorporated to the registry. A registry module for collecting the data regarding myocardial infarctions (MIs), hospitalizations due to unstable angina, subsequent revascularizations, strokes, and other complications was under construction at the time of analysis.

\section{Data Collection}

A detailed questionnaire, defined according to standard definitions, including demographic data, history, physical findings, management, imaging studies, and outcomes, was developed. Data were collected either at presentation or by physician review of the hospital records and were forwarded to the KROK registry. The forms were reviewed for clinical face validity and analytical internal validity.

\section{Study Population and Clinical Variables}

Using the KROK participant user file, we identified patients undergoing MV surgery between 2006 and 2017. Of 34,267 MV surgery records, we excluded those without history of AF or AF at time of presentation. Both MV repairs and replacement surgeries were eligible; both MV stenosis and regurgitation could have served as an indication. Regurgitation underlying pathologies encompassed initially rheumatic, functional, degenerative, congenital, endocarditis, calcific, ischemic, and other causes of mitral regurgitation. To better corroborate the results as well as to follow the line of the current guidelines, these were retrospectively categorized into primary and secondary regurgitation according to pertinent definitions. ${ }^{26}$ 
For patients undergoing MV surgery, we considered and report 3 categories of variables as potentially influencing the primary end point: baseline demographic characteristics (age, gender, European system for cardiac operative risk evaluation [EuroSCORE] score, diabetes, body mass, hypertension, poor mobility, pulmonary hypertension, chronic kidney disease, vascular disease, chronic lung disease, left ventricle ejection fraction [LVEF], coronary artery disease [CAD], previous MI, previous percutaneous coronary intervention, Canadian Cardiovascular Society, and New York Heart Association functional class), MV pathology (stenosis, regurgitation, and regurgitation grade and category), and surgical characteristics (redo, endocarditis, cardiogenic shock, intra-aortic balloon pump [IABP] use, critical preoperative state, intravenous inotropes/nitrates, and concomitant procedures).

Primary end point assessed was late survival in MV + ablation group versus MV alone group. Analyses of early postoperative mortality $(<24$ hours) and in-hospital mortality were performed as well. Additionally, operative times, length of intensive care unit (ICU) stay, and hospital stay (HLoS) are reported.

\section{Statistical Analysis}

Continuous, normally distributed variables were summarized as mean \pm standard deviation. Variables with nonnormal distributions were summarized as median and interquartile range and compared with the Mann-Whitney $U$ test or standard $t$ test as appropriate. Categorical variables were expressed as number ( $\%$ ) and compared with the Fisher exact test. Cox proportional-hazards models were used to determine factors related to event-free survival. The ensuing statistical models were used to define the hazard ratios (HRs), point estimate, and $95 \%$ confidence interval $(95 \% \mathrm{CI})$ of the effect size and to evaluate the efficacy of ablation with respect to MV surgery. Respective HRs for the comparison of $\mathrm{MV}+$ ablation patients versus MV alone patients were calculated and reported first for the univariate Cox proportional-hazards model taking into account all sets of variables categorized by baseline demographic characteristics, mitral valve pathology, and surgical characteristics. Next, a multivariate model was built, again stratified on the 3 sets of variables. Interaction between univariate and multivariate variables was assessed with the use of the Cochran-Mantel-Haenszel test. The multivariate model was then tested for multicollinearity.

Propensity score analysis was performed to balance possible confounding between the 2 study groups with regard to selected variables to prevent any bias related to the initial selection of patients for MV surgery. Propensity scores were computed using a multiple logistic regression model in which the dependent variable was concomitant ablation and the independent variables were those for which the given variable returned an estimated effect $\geq 0.1$ change in respective HR after multiple logistic regression. Regression adjustment was then fitted, resulting in increased precision for continuous outcome as described by Steyerberg. ${ }^{27}$ A greedy match using nearest-neighbor method was used and 1 to- 1 ratio, without replacement, within a specific caliper width of 0.2 standard deviations of the logit of the estimated propensity score. Propensity scores along with Wald $\chi^{2}$ test results are reported with corresponding $95 \%$ CIs. To verify the balance between the MV + ablation versus and MV alone groups after propensity score matching, the standardized mean differences were computed and were $<0.1$ in all cases. For the selected propensity scorematched population, univariate and multivariate Cox proportional hazard models were tested again and statistical differences reported. Overall late mortality was assessed with Kaplan-Meier curves fitted before (unadjusted model) and after propensity score matching. As a sensitivity analysis to assess the survival after concomitant ablation, patients were stratified according to defined subgroups and respective models unadjusted and propensity score match redone. STATA MP version 13.0 software (StataCorp, College Station, Tex) was used for computations.

\section{RESULTS}

During the 12-year study period, 34,267 patients undergoing MV surgery were identified. Among them 11,381 initially presented with AF. Subjects were divided into

Retrospective review 2007-2017

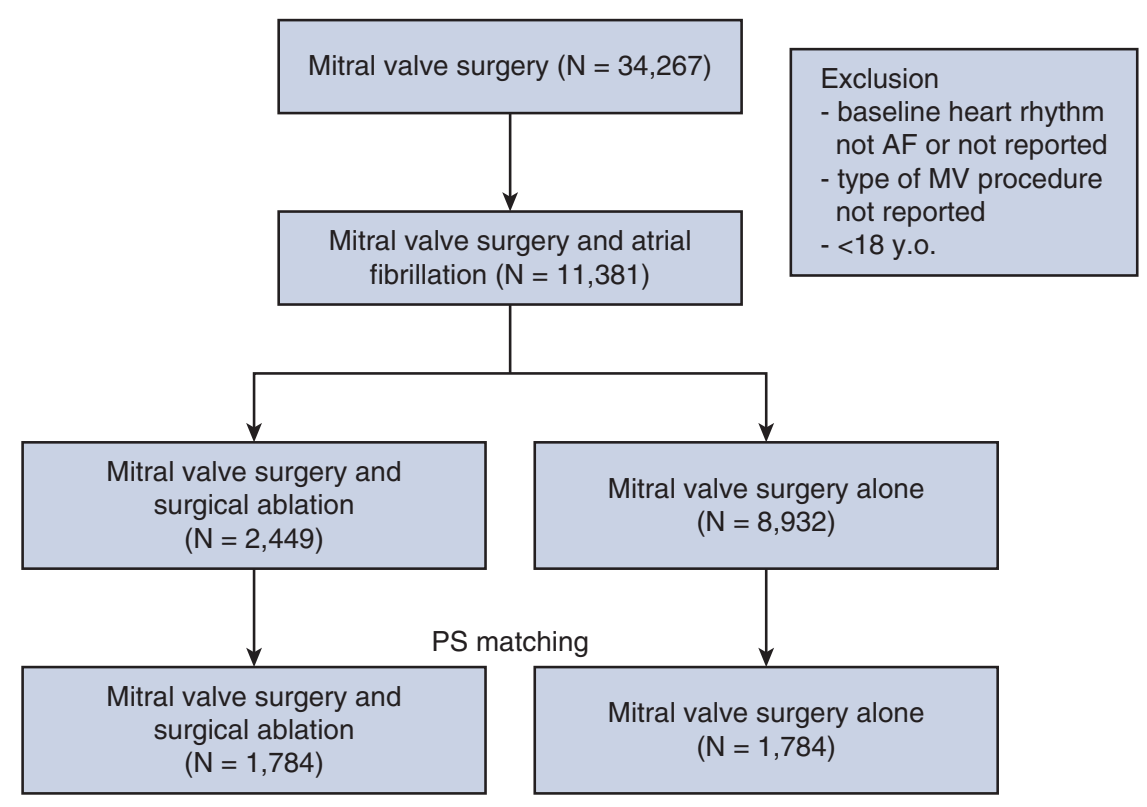

Median follow-up 4.6 years (max. 12.0 years)

FIGURE 1. Flow diagram of the current study cohort undergoing mitral valve $(M V)$ surgery with or without concomitant surgical ablation for atrial fibrillation $(A F)$. 
MV + ablation (2449 [21.52\%]) and MV alone (8932 [78.48\%]) groups (see Figure 1). Median follow-up was 5 years (mean, 4.6 years; interquartile range, 1.9-7.9 years). Baseline characteristics along with clinical and surgical data are listed in Table 1. Patients in the MV + ablation group were significantly younger $(63.8 \pm 8.7$ years vs $66.1 \pm 9.0$ years; $P<.001)$ and were at lower baseline surgical risk (mean EuroSCORE, 2.86 vs 3.69; $P<.001$ ). Despite similar percentage distribution of patients across ranges of Canadian Cardiovascular Society and New York Heart Association functional classes and LVEF, MV + ablation patients less often had previous MI $(10.0 \%$ vs $15.4 \%)$ and underwent percutaneous coronary intervention $(4.5 \%$ vs $6.8 \%)$. Regarding clinical characteristics at time of procedure, MV + ablation patients less commonly received intravenous inotropes $(0.6 \%$ vs $3.3 \% ; P<.001)$, or had an IABP inserted $(0.4 \%$ vs $0.9 \% ; P=.003)$ compared with the MV alone subgroup.

Primary mitral regurgitation was reported nearly twice as often in the entire population as secondary regurgitation $(65.2 \%$ vs $31.0 \% ; P<.001)$ and the proportion was maintained in subgroup comparison of $\mathrm{MV}+$ ablation versus MV alone $(62.4 \%$ vs $66.0 \% ; P=.001)$ and for primary and secondary mitral regurgitation $(33.9 \%$ vs $31.2 \%$; $P=.011)$. Significantly more moderate mitral regurgitation was observed in patients undergoing MV + ablation than MV alone $(40.3 \%$ vs $30.3 \% ; P<.001)$. The details on operative data are presented in Table 2.

\section{Operative and Long-Term Data}

Significantly more patients received MV replacement than MV repair $6843(60.1 \%)$ versus 4538 (39.9\%); $P<.001$. In the subgroup analysis, MV replacement was significantly more frequent in the MV alone group $(62.1 \%$ vs $57.4 \% ; P<.001)$.

Overall average cardiopulmonary bypass time was $128 \pm 54$ minutes and was 10 minutes shorter in the MV alone group than in the MV + ablation group (125 \pm 53 minutes vs $136 \pm 55$ minutes; $P<.001)$. Similarly, average crossclamp time was $83 \pm 35$ minutes in the overall analysis and was 8 minutes shorter in the MV alone group than the MV + ablation group $(82 \pm 35$ minutes vs $90 \pm 35$ minutes; $P<.001$ ).

Average HLoS was $16 \pm 22$ days and ICU stay was $4.4 \pm 11.8$ days. There was no difference in either the duration of ICU stay between MV + ablation versus MV alone (4.4 days vs 4.4 days; $P=.941)$ or $\operatorname{HLoS}(P=.388)$.

There was a difference in early postoperative mortality between MV + ablation versus MV alone groups in favor of $\mathrm{MV}+$ ablation (risk ratio, 0.58 ; $95 \% \mathrm{CI}, 0.40-0.81$; $P=.005)$. In-hospital mortality was reduced as well (risk ratio, 0.72; 95\% CI, 0.60-0.88; $P<.001$ ). Within investigated follow-up, unadjusted HR for long-term survival favored surgical ablation (HR, $0.71 ; 95 \%$ CI 0.63 -
$0.79 ; P<.001$ ) (Figure 2). A list of remaining in-hospital outcomes is presented in Table 3.

\section{Propensity Score Analysis}

One-to-1 propensity score-matched analysis resulted in 1784 pairs with similar baseline characteristics and operative covariates (Tables 1 and 2). List of variables contributing to propensity score along with respective propensity scores is available in Table E1. Detailed analysis of standardized mean differences before and after propensity score matching comparing covariate values for patients undergoing MV + ablation versus MV alone (Figure E1) revealed that standard mean differences for the measured covariates were all $<0.1$, suggesting covariate balance across groups. Propensity score-matched late survival was estimated to HR 0.82; 95\% CI, 0.70-0.96; $P=.011$. (see Figure 2). Hospital outcomes adjusted for propensity score are reported in Table 3.

\section{Sensitivity and Subgroup Analyses}

A number of subgroup analyses were performed for comparison of MV + ablation versus MV alone before and after propensity score matching (Figures 3 and 4 ) with respect to the primary end point of long-term survival. In these analyses, several significant interactions with baseline (Figure 3) or procedure-related (Figure 4) variables were demonstrated. Before propensity score matching there was significantly higher extent of benefit in the MV + ablation subgroup of patients younger than age 50 years (HR, 0.36; 95\% CI, 0.16-0.81; $P=.013$ ); with EuroSCORE score of 2 through 5 (HR, 0.42; 95\% CI, $0.31-0.59 ; P<.001$ ), without pulmonary hypertension (HR, 0.44; 95\% CI, 0.31-0.62; $P<.001$ ), and in those without signs of heart failure (HR, $0.39 ; 95 \%$ CI, 0.19 $0.81 ; P=.012)$. In contrast, there was no evident benefit with ablation in patients presenting with poor mobility (HR, $1.29 ; 95 \% \mathrm{CI}, 0.74-2.27 ; P=.371$ ), pulmonary hypertension (HR, 1.13; 95\% CI, 0.84-1.51; $P=.432$ ), or decreased LVEF (<20\%) (HR, 1.13; 95\% CI, 0.53-2.42; $P=.750$ ). No significant interactions were observed in subgroup analyses stratified on procedure-related, mitral pathology, and surgery characteristics. Besides, the benefit of ablation was borderline less pronounced in patients undergoing a concomitant coronary artery bypass grafting procedure (HR, 0.81; 95\% CI, 0.66-1.00 vs HR, 0.63; $95 \%$ CI, 0.55-0.72; $P_{\text {Interaction }}=.049$ ).

After propensity score matching, the direction of benefit with concomitant ablation was maintained across all subgroups of patients, yet was particularly appraised in patients with lower baseline surgical risk, such as those with EuroSCORE score $<5$ and in those without diabetes, hypertension, kidney disease, IABP, intravenous inotropes, or endocarditis. 
TABLE 1. Preoperative characteristics before and after propensity score (PS) matching

\begin{tabular}{|c|c|c|c|c|c|c|c|c|}
\hline \multirow[b]{2}{*}{ Variable } & \multicolumn{4}{|c|}{ All patients } & \multicolumn{4}{|c|}{ PS-matched patients } \\
\hline & $\begin{array}{c}\text { Total } \\
(\mathrm{N}=\mathbf{1 1 , 3 8 1 )}\end{array}$ & $\begin{array}{c}\text { MV + ablation } \\
(\mathbf{n}=\mathbf{2 4 4 9})\end{array}$ & $\begin{array}{l}\text { MV alone } \\
(\mathbf{n}=\mathbf{8 9 3 2})\end{array}$ & $\begin{array}{c}P \\
\text { value }\end{array}$ & $\begin{array}{c}\text { Total } \\
(\mathrm{N}=\mathbf{3 5 6 8})\end{array}$ & $\begin{array}{c}\text { MV + ablation } \\
(\mathbf{n}=\mathbf{1 7 8 4})\end{array}$ & $\begin{array}{l}\text { MV alone } \\
(n=1784)\end{array}$ & $\begin{array}{c}P \\
\text { value }\end{array}$ \\
\hline Age (y) & $66.3(60.1-72.2)$ & $64.5(58.7-69.9)$ & $66.8(60.6-72.7)$ & $<.001$ & $65.2(60.2-71.1)$ & $64.9(60.1-70.8)$ & $65.3(60.2-71.0)$ & .427 \\
\hline$<50$ & $539(4.7)$ & $171(7.0)$ & $368(4.1)$ & $<.001$ & $118(3.3)$ & $65(3.6)$ & $57(3.2)$ & .461 \\
\hline $50-70$ & $6961(61.2)$ & $1674(68.4)$ & $5287(59.2)$ & $<.001$ & $1989(55.7)$ & $992(55.9)$ & $992(55.6)$ & .999 \\
\hline$>70$ & $3881(34.1)$ & $604(24.7)$ & $3277(36.7)$ & $<.001$ & $1461(40.9)$ & $722(40.5)$ & 739 (41.4) & .563 \\
\hline \multicolumn{9}{|l|}{ Gender } \\
\hline Male & $5298(46.6)$ & $1080(44.1)$ & $4218(47.2)$ & .006 & $1638(45.9)$ & $813(45.6)$ & $825(46.2)$ & .687 \\
\hline Female & $6083(53.4)$ & $1369(55.9)$ & $4714(52.8)$ & .006 & $1930(54.1)$ & $971(54.4)$ & $959(53.8)$ & .687 \\
\hline EuroSCORE & $1.65(0.96-3.64)$ & $1.46(0.88-3.23)$ & $1.70(0.99-3.78)$ & $<.001$ & $1.55(0.93-3.42)$ & $1.52(0.91-3.48)$ & $1.57(0.96-3.41)$ & .197 \\
\hline$<2$ & $6413(56.4)$ & $1479(60.4)$ & $4934(55.2)$ & $<.001$ & $2088(58.5)$ & $1039(958.2)$ & $1049(58.8)$ & .734 \\
\hline $2-5$ & $2970(26.1)$ & $631(25.8)$ & $2339(26.2)$ & .674 & $904(25.3)$ & 457 (25.6) & $447(25.1)$ & .700 \\
\hline$>5$ & $1998(17.8)$ & 340 (13.9) & $1658(18.6)$ & $<.001$ & $576(16.1)$ & $288(16.1)$ & $288(16.1)$ & .999 \\
\hline Diabetes & 2689 (23.6) & $484(19.8)$ & $2205(24.7)$ & $<.001$ & $587(16.9)$ & $293(16.4)$ & $294(16.5)$ & .964 \\
\hline Insulin dependent & $858(7.5)$ & $154(6.3)$ & $704(7.9)$ & .008 & $245(6.9)$ & $116(6.5)$ & $129(7.2)$ & .389 \\
\hline Smoking & $5146(45.2)$ & $1120(45.7)$ & $4026(45.1)$ & .562 & $1996(55.9)$ & $992(55.6)$ & $1004(56.3)$ & .686 \\
\hline Hypertension & $8023(70.5)$ & $1595(65.1)$ & $6428(71.9)$ & $<.001$ & $1076(30.2)$ & $535(30.0)$ & $542(30.3)$ & .827 \\
\hline Poor mobility & $389(3.4)$ & $62(2.5)$ & $327(3.7)$ & .007 & $67(1.9)$ & $29(1.6)$ & $38(2.1)$ & .266 \\
\hline Pulmonary hypertension & $1737(15.3)$ & 408 (16.7) & $1330(14.9)$ & .031 & $799(22.4)$ & 408 (22.9) & $391(21.9)$ & .292 \\
\hline $\begin{array}{c}\text { Moderate (PA systolic } \\
31-55 \mathrm{~mm} \mathrm{Hg})\end{array}$ & $1215(70.0)$ & $309(75.6)$ & $906(68.1)$ & .003 & $559(70.0)$ & $288(70.1)$ & $273(69.8)$ & .490 \\
\hline $\begin{array}{l}\text { Severe (PA systolic } \\
\quad>55 \mathrm{~mm} \mathrm{Hg})\end{array}$ & $523(30.0)$ & $99(24.4)$ & $424(31.9)$ & .003 & $220(30.0)$ & $120(29.9)$ & $118(30.2)$ & .893 \\
\hline Renal impairment & $3129(27.5)$ & $625(25.5)$ & $2505(28.0)$ & .013 & $741(20.7)$ & $369(20.6)$ & $372(20.8)$ & .942 \\
\hline $\begin{array}{l}\text { Moderate }(\mathrm{CC} \\
>50 \text { and }<85)\end{array}$ & $2276(72.7)$ & $497(79.5)$ & 1779 (71.0) & $<.001$ & $593(80.0)$ & $290(78.5)$ & $303(81.4)$ & .559 \\
\hline Severe $(C C<50)$ & $817(26.1)$ & $125(20.0)$ & $692(27.6)$ & $<.001$ & 145 (19.5) & $79(21.4)$ & $66(17.7)$ & .270 \\
\hline $\begin{array}{l}\text { Dialysis (regardless } \\
\text { of } \mathrm{CC} \text { ) }\end{array}$ & $37(1.2)$ & $3(0.5)$ & $34(1.4)$ & .077 & $3(0.5)$ & $1(0.1)$ & $2(0.5)$ & .571 \\
\hline Vascular disease & $1746(15.3)$ & $305(12.5)$ & $1441(16.1)$ & $<.001$ & $723(20.2)$ & $359(20.1)$ & $364(20.4)$ & .835 \\
\hline Extracardiac arteriopathy & $1240(10.9)$ & $196(8.0)$ & $1044(11.7)$ & $<.001$ & $291(8.2)$ & $133(7.5)$ & $158(8.9)$ & .127 \\
\hline History of CVA & $722(6.3)$ & $130(5.3)$ & $585(6.6)$ & .025 & $245(6.9)$ & $129(7.2)$ & $116(6.5)$ & .387 \\
\hline Chronic lung disease & $931(8.2)$ & $180(7.4)$ & $750(8.4)$ & .094 & $294(8.2)$ & $148(8.3)$ & $146(8.2)$ & .903 \\
\hline Asthma & $445(3.9)$ & $91(3.7)$ & $353(4.0)$ & .593 & $98(2.7)$ & $45(2.5)$ & $53(2.9)$ & .413 \\
\hline LVEF (\%) & $50(40-60)$ & $50(45-60)$ & $50(40-60)$ & $<.001$ & $50(40-60)$ & $50(40-60)$ & $50(40-60)$ & .746 \\
\hline$<20$ & $199(2.0)$ & $29(1.4)$ & $170(2.2)$ & .013 & $57(1.6)$ & $26(1.5)$ & $31(1.7)$ & .505 \\
\hline $21-30$ & 837 (8.6) & $123(5.8)$ & $714(9.4)$ & $<.001$ & $53(1.5)$ & $25(1.4)$ & $28(1.6)$ & .967 \\
\hline $31-50$ & $4385(45.1)$ & 947 (44.7) & $3438(45.2)$ & .679 & 1413 (39.6) & $711(39.9)$ & $702(39.3)$ & .758 \\
\hline$>50$ & $4307(44.3)$ & $1021(48.1)$ & $3286(43.2)$ & $<.001$ & $2045(57.3)$ & $1022(57.3)$ & $1023(57.3)$ & .973 \\
\hline CAD & $3055(26.8)$ & $528(21.5)$ & 2558 (28.6) & $<.001$ & 729 (20.4) & $353(19.8)$ & $376(21.1)$ & .339 \\
\hline $1 \mathrm{VD}$ & $1133(36.7)$ & $221(41.8)$ & 912 (35.7) & .013 & $353(48.4)$ & $180(51.0)$ & $173(46.0)$ & .6947 \\
\hline $2 \mathrm{VD}$ & 878 (28.5) & $158(30.0)$ & $720(28.1)$ & .508 & $175(24.0)$ & $78(22.1)$ & $97(25.8)$ & .165 \\
\hline $3 \mathrm{VD}$ & $1044(33.8)$ & $149(28.1)$ & $895(35.0)$ & .001 & 201 (27.6) & 95 (26.9) & $106(28.2)$ & .140 \\
\hline Previous MI & $1617(14.2)$ & $245(10.0)$ & $1372(15.4)$ & $<.001$ & $358(10.0)$ & $179(10.0)$ & $179(10.0)$ & .999 \\
\hline$>1$ & $349(3.1)$ & $57(2.3)$ & $292(3.3)$ & .017 & $52(1.5)$ & $27(1.5)$ & $25(1.5)$ & .780 \\
\hline Previous PCI & $721(6.3)$ & $111(4.5)$ & $610(6.8)$ & $<.001$ & $200(5.6)$ & $100(5.6)$ & $100(5.6)$ & .999 \\
\hline \multicolumn{9}{|l|}{$\mathrm{CCS}$} \\
\hline 0 & 3589 (31.5) & 895 (36.5) & $2694(30.2)$ & $<.001$ & $1203(33.7)$ & $601(33.7)$ & $602(33.7)$ & .971 \\
\hline 1 & 3358 (29.5) & $742(30.3)$ & $2616(29.3)$ & .354 & $1039(29.1)$ & $526(29.5)$ & $513(28.8)$ & .632 \\
\hline 2 & $3046(26.8)$ & $567(23.2)$ & $2479(27.8)$ & $<.001$ & $910(25.5)$ & $440(24.7)$ & $470(26.3)$ & .249 \\
\hline
\end{tabular}


TABLE 1. Continued

\begin{tabular}{|c|c|c|c|c|c|c|c|c|}
\hline \multirow[b]{2}{*}{ Variable } & \multicolumn{4}{|c|}{ All patients } & \multicolumn{4}{|c|}{ PS-matched patients } \\
\hline & $\begin{array}{c}\text { Total } \\
(\mathrm{N}=\mathbf{1 1 , 3 8 1})\end{array}$ & $\begin{array}{c}\text { MV + ablation } \\
(\mathrm{n}=\mathbf{2 4 4 9 )}\end{array}$ & $\begin{array}{l}\text { MV alone } \\
(n=8932)\end{array}$ & $\begin{array}{c}P \\
\text { value }\end{array}$ & $\begin{array}{c}\text { Total } \\
(\mathbf{N}=\mathbf{3 5 6 8})\end{array}$ & $\begin{array}{c}\text { MV + ablation } \\
(\mathbf{n}=\mathbf{1 7 8 4})\end{array}$ & $\begin{array}{l}\text { MV alone } \\
(\mathrm{n}=1784)\end{array}$ & $\begin{array}{c}P \\
\text { value }\end{array}$ \\
\hline 3 & $1174(10.3)$ & $217(8.9)$ & $957(10.7)$ & .008 & $360(10.1)$ & $188(10.5)$ & $172(9.6)$ & .374 \\
\hline 4 & $214(1.9)$ & $28(1.1)$ & $186(2.1)$ & .003 & $56(1.6)$ & $29(1.6)$ & $27(1.5)$ & .788 \\
\hline \multicolumn{9}{|c|}{ NYHA functional class } \\
\hline 0 & $187(1.7)$ & $45(1.8)$ & $142(1.6)$ & .402 & $37(1.0)$ & $19(1.1)$ & $18(1.0)$ & .879 \\
\hline I & $567(5.0)$ & $136(5.6)$ & $431(4.9)$ & .137 & $176(4.9)$ & $82(4.6)$ & $94(5.3)$ & .165 \\
\hline II & $3605(31.9)$ & $850(35.1 \%)$ & $2755(31.0)$ & $<.001$ & $1155(32.4)$ & $568(31.8 \%)$ & $587(32.9)$ & .497 \\
\hline III & $5769(51.0)$ & $1238(51.0)$ & $4531(51.0)$ & .969 & $1942(54.4)$ & $963(54.0)$ & $979(54.9)$ & .141 \\
\hline IV & $1180(10.4)$ & $156(6.5)$ & $1024(11.5)$ & $<.001$ & $266(7.5)$ & $142(8.0)$ & $124(7.0)$ & .329 \\
\hline
\end{tabular}

Values are presented as median (interquartile range) or n (\%). PS, Propensity score; $M V$, mitral valve; EuroSCORE, European system for cardiac operative risk evaluation; $P A$, pulmonary artery; $C C$, creatinine clearance; $C V A$, cardiovascular accident; $L V E F$, left ventricle ejection fraction; $C A D$, coronary artery disease; $V D$, vessel disease; MI, myocardial infarction; PCI, percutaneous coronary intervention; CCS, Canadian Cardiovascular Society; NYHA, New York Heart Association.

The detailed analysis with reporting for both univariate and multivariate Cox proportional hazards model is presented in Table E2.

\section{DISCUSSION}

$\mathrm{AF}$, which is common among patients undergoing MV surgery, is a risk factor for death and morbidity. ${ }^{5-9} \mathrm{AF}$ correction performed at the time of index MV surgery procedure is safe and effective in restoring sinus rhythm, as reflected in the current American guidelines, ${ }^{21}$ which assigned Class I, Level A-recommendation stating that surgical ablation for AF can be performed without additional risk of operative mortality or major morbidity. European Society of Cardiology/European Society of Cardiothoracic Surgery guidelines recommend surgical ablation in patients undergoing cardiac surgery to improve symptoms attributable to AF, balancing the added risk of the procedure and the benefit of rhythm control therapy. A patient's high

TABLE 2. Operative characteristics before and after propensity score (PS) matching

\begin{tabular}{|c|c|c|c|c|c|c|c|c|}
\hline \multirow[b]{2}{*}{$\begin{array}{l}\text { Procedure-related } \\
\text { characteristic }\end{array}$} & \multicolumn{4}{|c|}{ All patients } & \multicolumn{4}{|c|}{ PS-matched patients } \\
\hline & $\begin{array}{c}\text { Total } \\
(\mathrm{N}=\mathbf{1 1}, \mathbf{3 8 1})\end{array}$ & $\begin{array}{c}\text { MV + ablation } \\
(\mathrm{n}=\mathbf{2 4 4 9})\end{array}$ & $\begin{array}{l}\text { MV alone } \\
(\mathrm{n}=\mathbf{8 9 3 2})\end{array}$ & $\begin{array}{c}P \\
\text { value }\end{array}$ & $\begin{array}{c}\text { Total } \\
(N=3568)\end{array}$ & $\begin{array}{c}\text { MV + ablation } \\
(\mathrm{n}=\mathbf{1 7 8 4})\end{array}$ & $\begin{array}{l}\text { MV alone } \\
(n=1784)\end{array}$ & $\begin{array}{c}P \\
\text { value }\end{array}$ \\
\hline Redo surgery & $966(8.5)$ & $64(2.9)$ & $902(10.1)$ & $<.001$ & $182(5.1)$ & $91(5.1)$ & $91(5.1)$ & .818 \\
\hline Endocarditis & $379(3.3)$ & $28(1.2)$ & $351(3.9)$ & $<.001$ & $147(4.1)$ & $71(3.9)$ & $76(4.3)$ & .674 \\
\hline Cardiogenic shock & $127(1.1)$ & $4(0.2)$ & $123(1.4)$ & $<.001$ & $6(0.2)$ & $2(0.1)$ & $4(0.2)$ & .423 \\
\hline Critical preoperative state & $233(2.0)$ & $16(0.7)$ & $217(2.4)$ & $<.001$ & $28(0.8)$ & $12(0.7)$ & $16(0.8)$ & .449 \\
\hline IABP & $92(0.8)$ & $8(0.4)$ & $84(0.9)$ & .003 & $16(0.4)$ & $8(0.4)$ & $8(0.4)$ & .999 \\
\hline Intravenous inotropes & $313(2.7)$ & $14(0.6)$ & $299(3.3)$ & $<.001$ & $30(0.8)$ & $13(0.7)$ & $17(0.8)$ & .465 \\
\hline Intravenous nitrates & $1087(9.6)$ & $316(14.0)$ & $771(8.6)$ & $<.001$ & $276(7.7)$ & $138(7.7)$ & $138(7.7)$ & .999 \\
\hline \multicolumn{9}{|l|}{ Mitral pathology } \\
\hline \multicolumn{9}{|l|}{ Mitral regurgitation grade } \\
\hline Trivial & $578(5.1)$ & $81(3.6)$ & $497(5.6)$ & $<.001$ & $165(4.6)$ & $79(4.4)$ & $86(4.8)$ & .436 \\
\hline Mild & $1067(9.4)$ & $247(11.0)$ & $821(9.2)$ & .179 & $321(8.9)$ & $172(9.6)$ & $149(8.3)$ & .178 \\
\hline Moderate & $3614(31.8)$ & $907(40.3)$ & $2708(30.3)$ & $<.001$ & $1001(28.0)$ & $492(27.5)$ & $509(28.5)$ & .526 \\
\hline Severe & $5607(49.3)$ & $1113(49.5)$ & $4495(50.3)$ & $<.001$ & $1890(52.9)$ & $940(52.6)$ & $950(53.2)$ & .737 \\
\hline \multicolumn{9}{|l|}{ Mitral pathology } \\
\hline Primary & $7425(65.2)$ & $1527(62.4)$ & $5898(66.0)$ & .001 & 2497 (65.9) & $1254(70.3)$ & $1243(69.7)$ & .688 \\
\hline Secondary & $3615(31.8)$ & 830 (33.9) & 2785 (31.2) & .011 & 1068 (29.9) & 534 (29.9) & 534 (29.9) & .999 \\
\hline Mitral valve stenosis & $3261(28.7)$ & $721(32.0)$ & $2540(28.4)$ & .331 & $1020(28.6)$ & $510(28.6)$ & $510(28.6)$ & .999 \\
\hline \multicolumn{9}{|l|}{ Surgery } \\
\hline MV replacement & $6843(60.1)$ & $1292(57.4)$ & $5551(62.1)$ & $<.001$ & $2140(59.9)$ & $1070(59.9)$ & $1070(59.9)$ & .999 \\
\hline $\mathrm{MV}+\mathrm{CABG}$ & $2621(23.0)$ & $459(20.4)$ & $2161(24.2)$ & $<.001$ & 819 (22.9) & $414(23.2)$ & 405 (22.7) & .720 \\
\hline $\mathrm{MV}+\mathrm{AV}$ & $2145(18.9)$ & $400(17.8)$ & 1745 (19.5) & $<.001$ & $68(1.9)$ & 35 (1.9) & $33(1.8)$ & .806 \\
\hline $\mathrm{MV}+\mathrm{TV}$ & $4890(43.0)$ & $1190(52.9)$ & $3700(41.4)$ & $<.001$ & $1424(39.9)$ & $714(40.0)$ & 710 (39.7) & .891 \\
\hline
\end{tabular}

Values are presented as n (\%). $P S$, Propensity score; $M V$, mitral valve; $I A B P$, intra-aortic balloon pump; $C A B G$, coronary artery bypass grafting; $A V$, aortic valve; $T V$, tricuspid valve. 


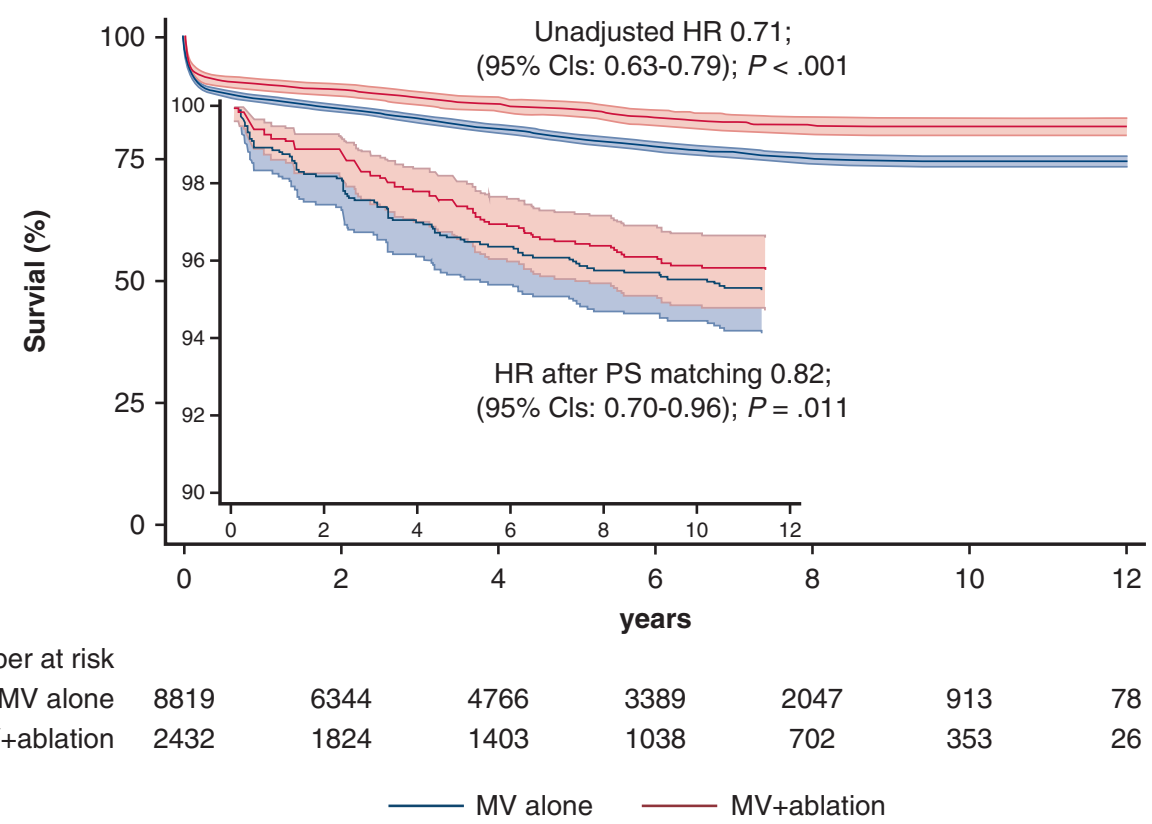

FIGURE 2. Unadjusted and propensity score $(P S)$-matched Kaplan-Meier survival curves between the 2 groups: mitral valve $(M V)+$ ablation versus MV alone. Hazard ratios $(H R s)$ and respective $95 \%$ confidence intervals $(C I s)$ in the $\mathrm{MV}+$ ablation compared with MV alone.

baseline risk and reoperation status occasionally triggers the decision not to perform ablation ${ }^{21,28}$ because it may prolong the cardiopulmonary bypass and crossclamp times. Yet, most of the available studies suggest that worse risk profiles are not a contraindication to surgical ablation and others found reduced mortality in high-risk patients. ${ }^{18}$ In this contradictory context, demonstration of a survival benefit in patients with mitral disease after surgical ablation has long been difficult. ${ }^{23,29}$

The above divergences may be due to limited sample sizes and follow-up duration in randomized controlled trials; indeed, the first Scandinavian randomized controlled trial of 69 patients with permanent AF undergoing MV surgery with or without epicardial left atrial cryoablation found

TABLE 3. In-hospital outcomes before and after propensity score (PS) matching

\begin{tabular}{|c|c|c|c|c|c|c|c|c|}
\hline & \multicolumn{4}{|c|}{ All patients } & \multicolumn{4}{|c|}{ PS-matched patients } \\
\hline & $\begin{array}{c}\text { MV + ablation } \\
(\mathbf{n}=\mathbf{2 4 4 9})\end{array}$ & $\begin{array}{l}\text { MV alone } \\
(\mathbf{n}=\mathbf{8 9 3 2})\end{array}$ & $\begin{array}{c}\text { Risk ratio } \\
(95 \% \text { confidence } \\
\text { interval) } \\
\end{array}$ & $P$ value & $\begin{array}{c}\text { MV + ablation } \\
(\mathbf{n}=\mathbf{1 7 8 4})\end{array}$ & $\begin{array}{l}\text { MV alone } \\
(\mathbf{n}=\mathbf{1 7 8 4})\end{array}$ & $\begin{array}{c}\text { Risk ratio } \\
(95 \% \text { confidence } \\
\text { interval }) \\
\end{array}$ & $\begin{array}{c}P \\
\text { value }\end{array}$ \\
\hline Early postoperative mortality & $31(1.3)$ & $192(2.1)$ & $0.58(0.40-0.81)$ & .005 & $25(1.4)$ & $49(2.7)$ & $0.51(0.32-0.82)$ & .005 \\
\hline In-hospital mortality & $124(5.1)$ & $621(6.9)$ & $0.72(0.60-0.88)$ & $<.001$ & $98(5.5)$ & $138(7.7)$ & $0.71(0.55-0.91)$ & .007 \\
\hline $\begin{array}{l}\text { Cardiac tamponade and/or } \\
\text { rethoracotomy }\end{array}$ & $290(11.8)$ & $1031(11.5)$ & $1.03(0.91-1.16)$ & .682 & $220(12.3)$ & $243(13.6)$ & $0.90(0.76-1.07)$ & .252 \\
\hline Periprocedural MI & $11(0.4)$ & $64(0.7)$ & $0.63(0.33-1.19)$ & .151 & $11(0.6)$ & $15(0.8)$ & $0.73(0.34-1.59)$ & .434 \\
\hline Respiratory failure & $216(8.8)$ & $795(8.9)$ & $0.99(0.86-1.14)$ & .901 & 209 (11.7) & $214(12.0)$ & $0.98(0.82-1.17)$ & .796 \\
\hline Prolonged ICU stay & $52(2.1)$ & $146(1.6)$ & $1.30(0.95-1.78)$ & .102 & $34(1.9)$ & $43(2.4)$ & $0.9(0.51-1.23)$ & .301 \\
\hline Neurologic complications & $72(2.9)$ & $313(3.5)$ & $0.84(0.65-1.08)$ & .172 & $65(3.6)$ & $89(5.0)$ & $0.73(0.53-0.99)$ & .049 \\
\hline Multiogran failure & $77(3.1)$ & $358(4.0)$ & $0.78(0.62-1.00)$ & .055 & $58(3.3)$ & $91(5.1)$ & $0.64(0.46-0.88)$ & .006 \\
\hline $\begin{array}{r}\text { Gastrointestinal } \\
\text { complications }\end{array}$ & $27(1.1)$ & $146(1.6)$ & $0.67(0.45-1.01)$ & .059 & $25(1.4)$ & $32(1.8)$ & $0.78(0.46-1.31)$ & .351 \\
\hline Acute kidney failure & $65(2.7)$ & $273(3.1)$ & $0.87(0.67-1.13)$ & .300 & $54(3.0)$ & $57(3.2)$ & $0.95(0.66-1.37)$ & .772 \\
\hline Sternal wound infection & $30(1.2)$ & $131(1.5)$ & $0.84(0.56-1.24)$ & .371 & $29(1.6)$ & $31(1.7)$ & $0.93(0.57-1.55)$ & .795 \\
\hline
\end{tabular}

Values are presented as n (\%), unless otherwise noted. $P S$, Propensity score; $M V$, mitral valve; $M I$, myocardial infarction; $I C U$, intensive care unit. 


\begin{tabular}{|c|c|c|c|c|c|}
\hline Variable & $\begin{array}{c}\text { Favors } \\
\leftarrow \text { MV+ablation vs MV alone } \rightarrow\end{array}$ & Hazard Ratios (95\% Cls) & $P_{\text {value }}{ }^{*}$ & $P_{\text {Incosistency }}{ }^{* *}$ & $P_{\text {Interaction }}{ }^{* \star *}$ \\
\hline \multicolumn{6}{|l|}{ Age } \\
\hline$<50$ & - & $0.79(0.50-1.26)$ & .321 & .098 & \multirow{3}{*}{.536} \\
\hline $50-70$ & $\square$ & $0.91(0.86-0.98)$ & .007 & .001 & \\
\hline$>70$ & & $0.83(0.68-1.01)$ & .057 & .764 & \\
\hline \multicolumn{6}{|l|}{ Gender } \\
\hline Male & $-\square$ & $0.89(0.82-0.97)$ & .007 & $<.001$ & \multirow{2}{*}{.223} \\
\hline Female & 品 & $0.96(0.88-1.05)$ & .365 & .001 & \\
\hline \multicolumn{6}{|l|}{ EuroSCORE } \\
\hline$<2$ & & $0.88(0.81-0.96)$ & .003 & .003 & \multirow{3}{*}{.016} \\
\hline$>2<5$ & - & $0.87(0.81-0.94)$ & $<.001$ & $<.001$ & \\
\hline$>5$ & & $1.11(0.95-1.29)$ & .181 & .072 & \\
\hline \multicolumn{6}{|l|}{ Diabetes } \\
\hline No diabetes/diet & & $0.89(0.83-0.96)$ & .002 & $<.001$ & \multirow{3}{*}{.112} \\
\hline Oral antihyperglycemic drugs & $\rightarrow-$ & $1.09(0.89-1.33)$ & .400 & .003 & \\
\hline Diabetes on insulin & & $1.03(0.81-1.31)$ & .810 & .192 & \\
\hline \multicolumn{6}{|l|}{ Smoking } \\
\hline Yes & $\square$ & $0.87(0.80-0.96)$ & .005 & .018 & \multirow{2}{*}{.148} \\
\hline No & $x_{2}$ & $0.96(0.88-1.04)$ & .338 & $<.001$ & \\
\hline \multicolumn{6}{|l|}{ Hypertension } \\
\hline Yes & $\square$ & $0.94(0.87-1.02)$ & .127 & $<.001$ & \\
\hline No & - & $0.87(0.78-0.97)$ & .012 & .001 & .261 \\
\hline Poor mobility & & & & & \\
\hline Yes & & $0.79(0.55-1.14)$ & .205 & .151 & (2) \\
\hline No & $\square$ & $0.92(0.86-0.98)$ & .012 & .018 & .420 \\
\hline Pulmonary hypertension & & & & & \\
\hline Yes & & $0.90(0.83-0.98)$ & .013 & .143 & .076 \\
\hline No & $\rightarrow$ & $1.01(0.92-1.12)$ & .840 & $<.001$ & \\
\hline Chronic kidney disease & & & & & \\
\hline Yes & - 혀 & $0.84(0.68-1.04)$ & .108 & .383 & 369 \\
\hline No & 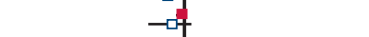 & $0.93(0.87-0.99)$ & .028 & .375 & .369 \\
\hline Vascular disease & & & & & \\
\hline Yes & $=0$ & $0.68(0.48-0.97)$ & .032 & .705 & \\
\hline No & (1) & $0.94(0.87-1.00)$ & .055 & $<.001$ & .081 \\
\hline Extracardiac arteriopathy & & & & & \\
\hline Yes & $\Rightarrow$ & $0.83(0.68-1.01)$ & .065 & .910 & ק \\
\hline No & 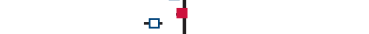 & $0.93(0.87-0.99)$ & .028 & $<.001$ & .284 \\
\hline Chronic lung disease & & & & & \\
\hline Yes & $\rightarrow-4$ & $0.99(0.79-1.24)$ & .930 & .078 & \\
\hline No & ( & $0.91(0.86-0.98)$ & .006 & $<.001$ & .504 \\
\hline LVEF & & & & & \\
\hline$<20 \%$ & $\Rightarrow$ & $0.79(0.50-1.26)$ & .317 & .430 & \\
\hline $20-30 \%$ & $\rightarrow \rightarrow$ & $0.90(0.71-1.14)$ & .383 & .273 & \\
\hline $30-50 \%$ & $\rightarrow$ & $0.87(0.79-0.97)$ & .012 & $<.001$ & $.5 / 5$ \\
\hline$>50 \%$ & 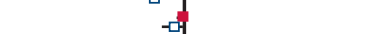 & $0.95(0.87-1.03)$ & .234 & .142 & \\
\hline CAD & & & & & \\
\hline No CAD & "أه. & $0.94(0.87-1.01)$ & .104 & .001 & \\
\hline $1 \mathrm{VD}$ & $-\square=$ & $0.97(0.77-1.22)$ & .795 & .094 & 235 \\
\hline 2 VD & $=$ & $0.87(0.66-1.16)$ & .333 & .886 & .235 \\
\hline 3 VD & $=$ =다 & $0.72(0.56-0.93)$ & .011 & .758 & \\
\hline Previous MI & & & & & \\
\hline Yes & - 며 & $0.87(0.80-0.96)$ & .004 & .299 & 152 \\
\hline No & $\square$ & $0.96(0.88-1.05)$ & .365 & $<.001$ & .152 \\
\hline Previous $\mathrm{PCl}$ & & & & & \\
\hline Yes & & $0.88(0.67-1.16)$ & .361 & .024 & \\
\hline No & 0 & $0.92(0.86-0.98)$ & .012 & $<.001$ & .757 \\
\hline $\operatorname{ccs}$ & & & & & \\
\hline No signs of angina & $-a$ & $0.94(0.84-1.04)$ & .229 & .002 & \\
\hline 1 & $-\rightarrow$ & $0.97(0.87-1.09)$ & .596 & $<.001$ & \\
\hline 2 & 量 & $0.85(0.75-0.97)$ & .013 & .857 & .111 \\
\hline 3 & -7) & $0.80(0.64-0.99)$ & .045 & .724 & \\
\hline 4 & 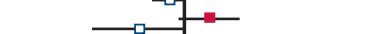 & $1.55(0.90-2.70)$ & .118 & .014 & \\
\hline NYHA & & & & & \\
\hline No signs of heart failure & $\Rightarrow$ & $0.81(0.46-1.45)$ & .472 & .839 & \\
\hline 1 & -7 & $1.11(0.83-1.49)$ & .484 & .009 & \\
\hline II & -1 & $0.98(0.88-1.10)$ & .723 & .066 & .638 \\
\hline III & - & $0.95(0.87-1.03)$ & .234 & .002 & \\
\hline IV & $\rightarrow-7$ & $0.87(0.72-1.04)$ & .138 & .020 & \\
\hline & 1 & गा & & & \\
\hline 0.01 & 0.1 & 100 & & & \\
\hline
\end{tabular}

FIGURE 3. Hazard ratios (HRs) and $95 \%$ confidence intervals $(C I s)$ for death from any cause in the mitral valve $(M V)+$ ablation group compared with MV alone group according to selected preoperative baseline characteristics. EuroSCORE, European system for cardiac operative risk evaluation; LVEF, left ventricle ejection fraction; $C A D$, coronary artery disease; VD, vessel disease; $M I$, myocardial infarction; $P C I$, percutaneous coronary intervention; $C C S$, Canadian Cardiovascular Society; $N Y H A$, New York Heart Association. $* P$ value for the treatment effect. $* * P$ value for the interaction between preand post propensity score $(P S)$ matching estimates. ${ }^{* * *} P$ value for the interaction between subgroup components (after PS matching). 


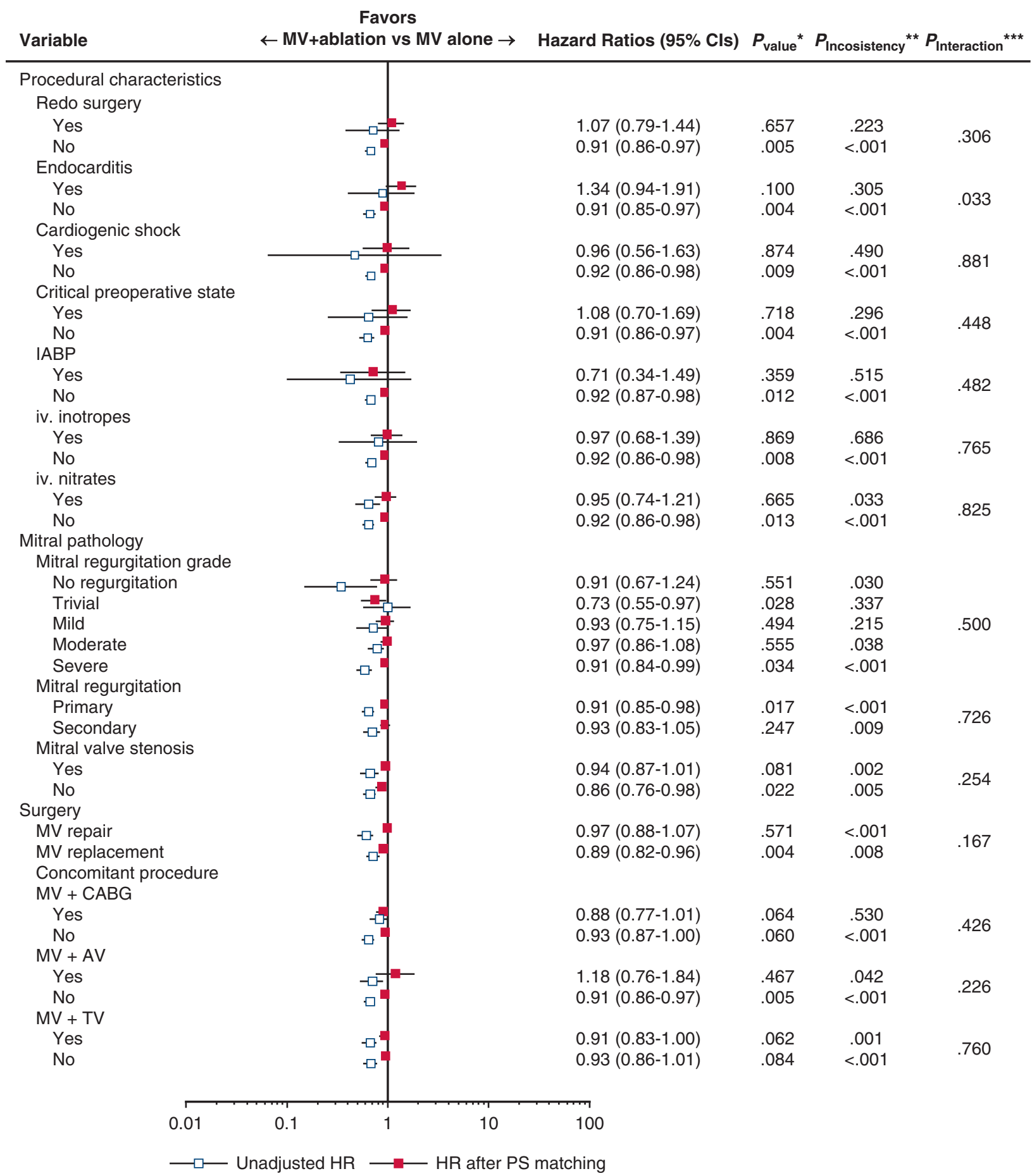

FIGURE 4. Hazard ratios (HRs) and $95 \%$ confidence intervals $(C I s)$ for death from any cause in the mitral valve ( $M V)+$ ablation group compared with MV alone group according to selected procedural characteristics. $I A B P$, Intra-aortic balloon pump; $i v$, intravenous; $C A B G$, coronary artery bypass grafting; $A V$, aortic valve; $T V$, tricuspid valve. ${ }^{*} P$ value for the treatment effect. $* * P$ value for the interaction between pre- and post propensity score $(P S)$ matching estimates. $* * * P$ value for the interaction between subgroup components (after PS matching).

65 patients reached the primary end point of regaining sinus rhythm. ${ }^{30}$ At 12 months' follow-up, maintenance rate was $73.3 \%$ but the study was underpowered for in-hospital complications and did not report longer than 1 year results. In a
Cardiothoracic Surgical Trials Network trial involving 260 patients with persistent or long-standing persistent $\mathrm{AF}$, the addition of surgical ablation at the time of MV surgery significantly increased the rate of freedom from $\mathrm{AF}$ at 
1 year. ${ }^{4}$ Risk of major cardiac or cerebrovascular adverse events at 1 year was not different (HR, 0.76; 95\% CI, $0.32-1.84 ; P=.55$ ) but ablation was associated with a 2.6-fold increased risk of implantation of a permanent pacemaker, which soon after became an argument against performing surgical AF corrections. A meta-analysis of 1900 patients from 22 randomized controlled trials ${ }^{31}$ found individuals undergoing surgical ablation were indeed more likely to need a pacemaker following the procedure ( $7 \%$ vs $4 \%$ [only 1.7 -fold increase]), whereas in the recent STS analysis of 4449 patients undergoing surgical ablation, ${ }^{32}$ permanent pacemaker implantation followed in $1.03 \%$, suggesting in turn center/operator success dependence.

In contrast to previous randomized controlled trials, several observational series with larger data sets have shown signs of remote benefits in patients undergoing MV surgery and concomitant ablation ${ }^{11-17}$ and in particular in patients at high risk; in fact, in the largest-to-date report from STS on multiple valve procedures ${ }^{18}$ (predicted mortality $13.1 \%$ for 3-valve surgeries), in patients undergoing arrhythmia ablation procedures ${ }^{16,32,33}$ in the aortic valve + MV group and aortic valve $+\mathrm{MV}+$ tricuspid valve procedures group, preoperative plus intraoperative models had lower predicted mortality risk than patients not receiving these procedures (odds ratio [OR], 0.85; 95\% CI, 0.73-0.98 and OR, 0.77; 95\% CI, 0.60-0.99, respectively, for aorticmitral and aortic-mitral-tricuspid valves). Nevertheless, lack of substantial but first of all, randomized data regarding the long-term success and potential clinical influence of surgical AF correction on hard clinical outcomes (eg, survival and stroke) remains among the most significant gaps in the current literature.

The current study focus was long-term survival after MV surgery in patients with AF undergoing concomitant surgical ablation. The main finding arising from this report is significant survival benefit in the MV + ablation subgroup. The benefit was further maintained after propensity matching and in a number of subgroup analyses performed to support the advantage of ablation across diverse clinical presentations at baseline but also across different surgical scenarios.

$\mathrm{KROK}$ is an ongoing, nationwide, multi-institutional registry of heart surgery procedures in Poland. It is among the oldest European heart surgery registries of its kind. There were almost 35,000 patients undergoing MV surgeries in Poland between 2006 and 2017 of whom 11,400 (33\%) presented with AF, which is in line also with most recent American observations. ${ }^{18,19}$ Exactly mirroring the overseas trends, ${ }^{28}$ we observed higher rates of MV replacements in the MV without ablation (62.1\%) compared with increasing rates of repairs in $\mathrm{MV}+$ ablation subsets $(52.6 \%)$. Another issue that becomes apparent is a significant underuse of ablation in the current analysis $(21.5 \%$ [2449 out of 11,381]). Although partially reflecting current trends, we find this phenomenon disturbing, given that a majority of patients included in both MV + ablation and MV alone groups were in fact at low risk (6413 [56.4\%] had EuroSCORE score $<2$ ). Self-explanatory is the fact that prior registry studies reported selection biases toward avoiding ablation in higher-risk patients ${ }^{33}$ given the complexity of adding an antiarrhythmic procedure, ${ }^{34,35}$ higher AF recurrence rates in some groups of patients, ${ }^{4}$ and above all time consumption. Indeed, reluctance to perform ablation may reflect concerns about prolonging crossclamp times. In our study, cardiopulmonary bypass time and crossclamp time were on average 10 and 8 minutes longer, respectively, in patients undergoing concomitant ablation. The concern of prolonging operative times is unjustified because there were no differences in ICU stay and HLoS. Ad and colleagues ${ }^{10}$ reported that addition of Cox-maze procedure to MV procedures-even with a high degree of complexity-did not increase operative risk and demonstrated acceptable rhythm success, reduced $\mathrm{AF}$ burden, and resulted in remarkably low stroke rates in the long-term. Baseline risk and underlying conditions will be clinicians' primary focus in the early postoperative course in higher-risk patients rather than ablation's success itself. It is therefore of great importance to force AF correction surgeries at the time of MV repair or replacement because these patients are more likely to benefit from ablation in the long-term in terms of survival.

It should be noted that individual surgeon experience and training influences significantly the results of long-term surgical ablation for AF, highlighting the potentially crucial role of education and training in increasing implementation and ablation success rates, and thus long-term survival. Ad and colleagues ${ }^{10}$ found addition of Cox-maze procedure to MV procedures - even with a high degree of complexitydid not raise the operative mortality; however, correlating with ablation success was surgeon experience (OR, 0.98; 95\% CI, 0.96-0.997; $P=.025)$. Apparent from Gillinov and colleagues ${ }^{4}$ and reflected in the STS guidelines ${ }^{21}$ is the certain need for appropriate training and education that should be completed before the performance of surgical ablation and we highly recommend surgeons who are new to surgical AF to be proctored by an experienced surgeon in 3 to 5 cases before performing surgical ablation alone.

To better reflect the effect of long-term survival benefit in the present report, hazard analyses were additionally performed across different patient populations. In an unadjusted analysis, significant benefit was still present in higher-risk patients such as those with diabetes, chronic kidney disease, CAD, and chronic obstructive pulmonary disease irrespective of MV underlying pathology. Neither benefit nor harm was demonstrated in subgroups of patients older than age 70 years, with poor mobility, reduced ejection fraction $(<20 \%)$, and pulmonary hypertension, suggesting these patients may not benefit from concomitant 
ablation to the extent of their healthier counterparts in terms of survival. The above trends were mostly retained after propensity score matching, suggesting more pronounced benefit with surgical ablation in lower-risk patients. Unlike Rankin and colleagues, ${ }^{18}$ we found that addition of aortic valve or tricuspid valve surgery did not influence the long-term survival between ablation and nonablation groups. Hence, the magnitude and direction of the estimate in favor of ablation was maintained in this group of patients without apparent differences. Proving the robustness and validity of our investigation, the results of subgroup analyses suggest convergent, albeit lower, benefit of ablation in higher burden of CAD, secondary mitral regurgitation and concomitant coronary artery bypass grafting. This finding justifies the role and relevance of ablation in ischemic heart disease as well. Nonsignificant interactions in the survival estimates after ablation were found as well in the comparisons of MV replacement versus MV repair $(P=.167)$ and MV regurgitation versus MV stenosis $(P=.254)$, in turn suggesting consistent effect across MV underlying pathologies.

\section{Limitations}

The current registry was not designed to assess in detail the effectiveness of concomitant ablation; therefore, conversions to sinus rhythm and maintenance rates are not available. In the current literature, these range from $90 \%$ to $97 \%$ in Cox-maze III and IV procedures, down to approximately $60 \%$ in less-established lesions sets and variable energy sources. ${ }^{\mathrm{E}, 29}$ A new module of the registry to collect data regarding rehospitalizations and other complications such as stroke is underway and will in the future allow for such analyses. We could not account for left atrial appendage closure rates and ablation durations, techniques, and immediate success rates because these were not obligatory to complete during registry conception.

Whether left atrial appendage may translate into reduced risk of stroke will be shown in ongoing studies, ${ }^{\mathrm{E} 2}$ but it can be assumed that with higher prevalence of left atrial appendage closures the number of strokes might drop as well as adding to survival benefits. Analysis of permanent pacemaker implantation was not performed because most patients requiring permanent pacemaker were transferred to cardiology departments while receiving temporary epicardial pacing. Because this is performed outside surgery departments, cardiology procedures following surgery were not introduced into the registry.

\section{CONCLUSIONS}

Concomitant surgical ablation for $\mathrm{AF}$ in patients undergoing MV procedures is safe and feasible. In an unadjusted analysis and after rigorous propensity matching, surgical ablation was associated with improved long-term survival as well.

\section{Conflict of Interest Statement}

Authors have nothing to disclose with regard to commercial support.

The KROK investigators include Lech Anisimowicz, MD, PhD; Krzysztof Bartuś, MD, PhD; Andrzej Biederman, MD, PhD; Dariusz Borkowski, MD; Mirosław Brykczyński, MD, PhD; Paweł Bugajski, MD, PhD; Paweł Cholewiński, MD; Romuald Cichoń, MD, PhD; Marek Cisowski, MD, PhD; Marek Deja, $\mathrm{MD}, \mathrm{PhD}$; Antoni Dziatkowiak, MD, PhD; Tadeusz Gburek, MD; Leszek Gryczko, MD; Ireneusz Haponiuk, MD, Piotr Hendzel, MD, PhD; Tomasz Hirnle, MD, PhD; Stanisław Jabłonka, MD, PhD; Krzysztof Jarmoszewicz, MD; Jarosław Jasiński, MD; Marek Jasiński MD, PhD; Ryszard Jaszewski, MD, PhD; Marek Jemielity, MD, PhD; Ryszard Kalawski, MD, $\mathrm{PhD}$; Bogusław Kapelak, MD, PhD; Jacek Kaperczak, MD; Maciej A. Karolczak, MD, PhD; Mariusz Kowalewski, MD; Michał Krejca, MD, PhD; Wojciech Kustrzycki, MD, PhD; Mariusz Kuśmierczyk, MD, PhD; Paweł Kwinecki, MD; Bohdan J. Maruszewski MD, PhD; Marcin Maruszewski, MD; Maurycy Missima, MD; Jacek J. Moll, MD, PhD; Wojciech Ogorzeja, MD; Jacek Pająk, MD; Szymon Pawlak, MD; Wojciech Pawliszak, MD; Edward Pietrzyk, MD; Grzegorz Religa, MD; Jan Rogowski, MD, PhD; Jacek Różański, MD, PhD; Jerzy Sadowski, MD, PhD; Girish Sharma, MD; Janusz Skalski, MD, PhD; Jacek Skiba, MD, PhD; Ryszard Stanisławski, MD; Jakub Staromłyński, MD; Janusz Stążka, MD, PhD; Piotr Stępiński, MD; Kazimierz Suwalski, MD, PhD; Piotr Suwalski MD, PhD; Zdzisław Tobota, MD; Łukasz Tułecki, MD; Kazimierz Widenka, MD, PhD; Michał Wojtalik, MD, PhD; Stanisław Woś, MD, PhD; Marian Zembala, MD, PhD; Michał Oskar Zembala, MD, PhD; and Piotr Żelazny, MD.

\section{References}

1. Wang H, Han J, Wang Z, Yin Z, Liu Z, Jin Y, et al. A prospective randomized trial of the cut-and-sew maze procedure in patients undergoing surgery for rheumatic mitral valve disease. J Thorac Cardiovasc Surg. 2018;155:608-17.

2. Pearman CM, Poon SS, Bonnett LJ, Haldar S, Wong T, Mediratta N, et al. Minimally invasive epicardial surgical ablation alone versus hybrid ablation for atrial fibrillation: a systematic review and meta-analysis. Arrhythm Electrophysiol Rev. 2017;6:202-9.

3. McClure GR, Belley-Cote EP, Jaffer IH, Dvirnik N, An KR, Fortin G, et al. Surgical ablation of atrial fibrillation: a systematic review and meta-analysis of randomized controlled trials. Europace. 2018;20:1442-50.

4. Gillinov AM, Gelijns AC, Parides MK, DeRose JJ Jr, Moskowitz AJ, Voisine P, et al. Surgical ablation of atrial fibrillation during mitral-valve surgery. $N$ Engl J Med. 2015;372:1399-409.

5. January CT, Wann LS, Alpert JS, Calkins H, Cigarroa JE, Cleveland JC, et al 2014 AHA/ACC/HRS guideline for the management of patients with atrial fibrillation: executive summary: a report of the American College of Cardiology/ American Heart Association Task Force on Practice Guidelines and the Heart Rhythm Society. Circulation. 2014;130:2071-104.

6. Calkins H, Kuck KH, Cappato R, Brugada J, Camm AJ, Chen SA, et al. 2012 HRS/EHRA/ECAS expert consensus statement on catheter and surgical ablation of atrial fibrillation: recommendations for patient selection, procedural techniques, patient management and follow-up, definitions, endpoints, and research trial design. Europace. 2012;14:528-606.

7. Saxena A, Dinh DT, Reid CM, Smith JA, Shardey GC, Newcomb AE. Does preoperative atrial fibrillation portend a poorer prognosis in patients undergoing isolated aortic valve replacement? A multicentre Australian study. Can J Cardiol. 2013;29:697-703.

8. Quader MA, McCarthy PM, Gillinov AM, Alster JM, Cosgrove DM III, Lytle BW, et al. Does preoperative atrial fibrillation reduce survival after coronary artery bypass grafting? Ann Thorac Surg. 2004;77:1514-22. 
9. Levy F, Rusinaru D, Marechaux S, Charles V, Peltier M, Tribouilloy C. Determinants and prognosis of atrial fibrillation in patients with aortic stenosis. Am J Cardiol. 2015;116:1541-6.

10. Ad N, Holmes SD, Massimiano PS, Rongione AJ, Fornaresio LM. Long-term outcome following concomitant mitral valve surgery and Cox maze procedure for atrial fibrillation. $J$ Thorac Cardiovasc Surg. 2018;155:983-94.

11. Doukas G, Samani NJ, Alexiou C, Oc M, Chin DT, Stafford PG, et al. Left atrial radiofrequency ablation during mitral valve surgery for continuous atrial fibrillation: a randomized controlled trial. JAMA. 2005;294:2323-9.

12. Abreu Filho CA, Lisboa LA, Dallan LA, Spina GS, Grinberg M, Scanavacca M, et al. Effectiveness of the maze procedure using cooled-tip radiofrequency ablation in patients with permanent atrial fibrillation and rheumatic mitral valve disease. Circulation. 2005;112:I20-5.

13. Barnett SD, Ad N. Surgical ablation as treatment for the elimination of atrial fibrillation: a meta-analysis. J Thorac Cardiovasc Surg. 2006;131:1029-35.

14. Cheng DC, Ad N, Martin J, Berglin EE, Chang BC, Doukas G, et al. Surgica ablation for atrial fibrillation in cardiac surgery: a meta-analysis and systematic review. Innovations (Phila). 2010;5:84-96.

15. Phan K, Xie A, La Meir M, Black D, Yan TD. Surgical ablation for treatment of atrial fibrillation in cardiac surgery: a cumulative meta-analysis of randomised controlled trials. Heart. 2014;100:722-30.

16. Damiano RJ Jr, Schwartz FH, Bailey MS, Maniar HS, Munfakh NA, Moon MR, et al. The Cox maze IV procedure: predictors of late recurrence. J Thorac Cardiovasc Surg. 2011;141:113-21.

17. Attaran S, Saleh HZ, Shaw M, Ward A, Pullan M, Fabri BM. Does the outcome improve after radiofrequency ablation for atrial fibrillation in patients undergoing cardiac surgery? A propensity-matched comparison. Eur J Cardiothorac Surg. 2012:41:806-10; discussion 810-1

18. Rankin JS, He X, O'Brien SM, Jacobs JP, Welke KF, Filardo G, et al. The Society of Thoracic Surgeons risk model for operative mortality after multiple valve surgery. Ann Thorac Surg. 2013;95:1484-90.

19. Badhwar V, Rankin JS, Ad N, Grau-Sepulveda M, Damiano RJ, Gillinov AM, et al. Surgical ablation of atrial fibrillation in the United States: trends and propensity matched outcomes. Ann Thorac Surg. 2017;104:493-500.

20. Gammie JS, Haddad M, Milford-Beland S, Welke KF, Ferguson TB Jr, O'Brien SM, et al. Atrial fibrillation correction surgery: lessons from the Society Of Thoracic Surgeons National Cardiac Database. Ann Thorac Surg. 2008;85: 909-14.

21. Badhwar V, Rankin JS, Damiano RJ Jr, Gillinov AM, Bakaeen FG, Edgerton JR, et al. The Society of Thoracic Surgeons 2017 clinical practice guidelines for the surgical treatment of atrial fibrillation. Ann Thorac Surg. 2017;103:329-41.

22. Ad N, Damiano RJ Jr, Badhwar V, Calkins H, La Meir M, Nitta T, et al. Expert consensus guidelines: examining surgical ablation for atrial fibrillation. J Thorac Cardiovasc Surg. 2017;153:1330-54.e1331.
23. Kirchhof P, Benussi S, Kotecha D, Ahlsson A, Atar D, Casadei B, et al. 2016 ESC guidelines for the management of atrial fibrillation developed in collaboration with EACTS. Eur Heart J. 2016;37:2893-962.

24. Wang J, Meng X, Li H, Cui Y, Han J, Xu C. Prospective randomized comparison of left atrial and biatrial radiofrequency ablation in the treatment of atrial fibrillation. Eur J Cardiothorac Surg. 2009:35:116-22.

25. Saint LL, Damiano RJ Jr, Cuculich PS, Guthrie TJ, Moon MR, Munfakh NA, et al. Incremental risk of the Cox-maze IV procedure for patients with atrial fibrillation undergoing mitral valve surgery. J Thorac Cardiovasc Surg. 2013;146:1072-7.

26. Harb SC, Griffin BP. Mitral valve disease: a comprehensive review. Curr Cardiol Rep. 2017;19:73.

27. Steyerberg EW. Clinical Prediction Models: A Practical Approach to Development, Validation, and Updating. New York: Springer; 2009.

28. Rankin JS, Grau-Sepulveda MV, Ad N, Damiano RJ Jr, Gillinov AM, Brennan JM, et al. Associations between surgical ablation and operative mortality after mitral valve procedures. Ann Thorac Surg. 2018;105:1790-6.

29. Chen Y, Maruthappu M, Nagendran M. How effective is unipolar radiofrequency ablation for atrial fibrillation during concomitant cardiac surgery? Interact Car diovasc Thorac Surg. 2012;14:843-7.

30. Blomstrom-Lundqvist C, Johansson B, Berglin E, Nilsson L, Jensen SM, Thelin S, et al. A randomized double-blind study of epicardial left atrial cryoablation for permanent atrial fibrillation in patients undergoing mitral valve surgery: the Swedish Multicentre Atrial Fibrillation study (SWEDMAF). Eur Heart J. 2007:28:2902-8.

31. Huffman MD, Karmali KN, Berendsen MA, Andrei AC, Kruse J, McCarthy PM, et al. Concomitant atrial fibrillation surgery for people undergoing cardiac surgery. Cochrane Database Syst Rev. 2016;CD011814.

32. Ad N, Suri RM, Gammie JS, Sheng S, O'Brien SM, Henry L. Surgical ablation of atrial fibrillation trends and outcomes in North America. J Thorac Cardiovasc Surg. 2012;144:1051-60.

33. Lee R, McCarthy PM, Wang EC, Vaduganathan M, Kruse J, Malaisrie SC, et al Midterm survival in patients treated for atrial fibrillation: a propensity-matched comparison to patients without a history of atrial fibrillation. J Thorac Cardiovasc Surg. 2012;143:1341-51; discussion 1350-1.

34. Cox JL, Schuessler RB, D’Agostino HJ Jr, Stone CM, Chang BC, Cain ME, et al The surgical treatment of atrial fibrillation. III. Development of a definitive surgical procedure. J Thorac Cardiovasc Surg. 1991;101:569-83.

35. Prasad SM, Maniar HS, Camillo CJ, Schuessler RB, Boineau JP, Sundt TM III, et al. The Cox maze III procedure for atrial fibrillation: long-term efficacy in patients undergoing lone versus concomitant procedures. J Thorac Cardiovasc Surg. 2003;126:1822-8.

Key Words: mitral valve, registry, surgical ablation, longterm results, atrial fibrillation 


\section{E-References}

E1. van Laar C, Geuzebroek GS, Hofman FN, Van Putte BP. The totally thoracoscopic left atrial maze procedure for the treatment of atrial fibrillation. Multimed Man Cardiothorac Surg. 2016;2016.
E2. Whitlock R, Healey J, Vincent J, Brady K, Teoh K, Royse A, et al. Rationale and design of the left atrial appendage occlusion study (laaos) iii. Ann Cardiothorac Surg. 2014;3:45-54.

Concomitant aortic valve surgery iv. Inotropes

iv. Nitrates NYHA 0

3 vessel coronary disease

2 vessel coronary disease

Endocarditis

Concomitant tricuspid valve surgery

Primary mitral regurgitation

NYHA I

Mitral Regurgitation III

Poor mobility

Previous MI

Pumonary Hypertension

Percutaneous Coronary Intervention

CCS 4

CCS 2

Mitral Regurgitation 0

Diabetes (insulin dependent)

Urgency

LVEF <20\%

Smoking

Reoperation

Intraaortic Balloon Pump

Critical Preoperatve State

LVEF > 50\%

Coronary Artery Bypass Grafting

CCS 1

Hypertension

Diabetes (oral hypoglicemic drugs)

1 vessel coronary disease

CCS 3

Chronic Kidney Disease NYHA II

Cardiogenic shock Chronic Obstructive Pulmonary Disease

Vascular disease

Emergency

NYHA III

Mitral Regurgitation II

LVEF 20-30\%

Diabetes (diet)

LVEF 30-50\%

NYHA IV

Elective

Mitral Regurgitation I

Mitral Regurgitation IV

EuroSCORE

Mitral valve replacement

no coronary disease

Gender

CCS 0

$\bullet$

. 
TABLE E1. Propensity scores

\begin{tabular}{|c|c|c|c|c|c|c|c|}
\hline Variable & Wald $/ \chi^{2}$ & Standard error & Score & $95 \%$ Confidence interval & Odds ratio & $95 \%$ Confidence interval & $P$ value \\
\hline $\mathrm{CCS}=0$ & 66.734 & 0.155 & -1.264 & -0.961 to -1.568 & 0.282 & 0.209 to 0.382 & .000 \\
\hline $\mathrm{NYHA}=0$ & 54.732 & 0.153 & -1.134 & -0.834 to -1.435 & 0.322 & 0.238 to 0.434 & .000 \\
\hline $\mathrm{CKD}=1$ & 44.955 & 0.151 & -1.013 & -0.717 to -1.309 & 1.363 & 1.270 to 1.488 & .000 \\
\hline $\mathrm{AV}=1$ & 13.305 & 0.157 & 0.574 & 0.866 to 0.282 & 1.563 & 1.414 to 0.767 & .000 \\
\hline $\mathrm{CABG}=1$ & 28.450 & 0.061 & 0.327 & 0.447 to 0.207 & 1.387 & 1.230 to 1.564 & .000 \\
\hline $\mathrm{COPD}=1$ & 19.505 & 0.081 & 0.359 & 0.519 to 0.200 & 1.432 & 1.221 to 1.680 & .000 \\
\hline $\mathrm{PCI}=1$ & 16.229 & 0.091 & 0.366 & 0.544 to 0.188 & 1.442 & 1.207 to 1.723 & .000 \\
\hline REDO $=1$ & 32.274 & 0.081 & 0.460 & 0.618 to 0.301 & 1.584 & 1.351 to 1.856 & .000 \\
\hline $\mathrm{LVEF}>50=1$ & 6.822 & 0.049 & 0.128 & 0.224 to 0.032 & 1.136 & 1.032 to 1.251 & .009 \\
\hline Insulin-dependent $\mathrm{T} 2 \mathrm{DM}=1$ & 4.065 & 0.088 & 0.178 & 0.351 to 0.005 & 1.195 & 1.005 to 1.421 & .044 \\
\hline $\mathrm{HT}=1$ & 5.923 & 0.054 & 0.132 & 0.239 to 0.026 & 1.141 & 1.026 to 1.270 & .015 \\
\hline Poor mobility $=1$ & 7.273 & 0.148 & 0.399 & 0.109 to 0.688 & 1.671 & 1.502 to 1.897 & .007 \\
\hline $\mathrm{PHT}=1$ & 347.024 & 0.049 & -0.914 & -0.818 to -1.010 & 1.401 & 1.364 to 1.441 & .000 \\
\hline
\end{tabular}

$C C S$, Canadian Cardiovascular Society; NYHA, New York Heart Association; $C K D$, chronic kidney disease; $A V$, aortic valve; $C A B G$, coronary artery bypass grafting; $C O P D$, chronic obstructive pulmonary disease; $P C I$, percutaneous coronary intervention; $R E D O$, reoperation; $L V E F$, left ventricle ejection fraction; $T 2 D M$, type 2 diabetes mellitus; $H T$, hypertension; $P H T$, pulmonary hypertension. 


\begin{tabular}{|c|c|c|c|c|c|c|c|c|c|c|}
\hline \multirow[b]{3}{*}{ Model } & \multicolumn{4}{|c|}{ Univariable } & \multicolumn{4}{|c|}{ Multivariable } & \multicolumn{2}{|c|}{$P$ for interaction } \\
\hline & \multicolumn{2}{|c|}{ Unadjusted hazard ratios } & \multicolumn{2}{|c|}{ After PS matching hazard ratios } & \multicolumn{2}{|c|}{ Unadjusted hazard ratios } & \multicolumn{2}{|c|}{ After PS matching hazard ratios } & \multirow{2}{*}{$\begin{array}{c}\text { Unadjusted } P \\
\text { interaction }\end{array}$} & \multirow{2}{*}{$\begin{array}{c}\text { After PS } \\
\text { matching } P \text { for } \\
\text { interaction }\end{array}$} \\
\hline & HR $(95 \%$ CI $)$ & $P$ value & HR $(95 \%$ CI $)$ & $P$ value & HR $(95 \%$ CI $)$ & $P$ value & HR $(95 \%$ CI $)$ & $P$ value & & \\
\hline \multicolumn{11}{|l|}{ Baseline characteristics } \\
\hline Age & $1.10(1.00-1.12)$ & .002 & $1.04(1.01-1.36)$ & .008 & - & - & - & - & - & - \\
\hline Gender & $1.06(1.04-1.12)$ & .006 & $0.87(0.68-0.97)$ & .013 & $0.85(0.82-0.89)$ & $<.001$ & - & - & .119 & - \\
\hline EuroSCORE & $1.02(1.01-1.02)$ & $<.001$ & $1.03(1.02-1.04)$ & $<.001$ & $1.06(1.06-1.07)$ & $<.001$ & $0.97(0.96-0.98)$ & $<.001$ & .089 & $<.001$ \\
\hline \multicolumn{11}{|l|}{ Diabetes } \\
\hline No diabetes/diet & $0.83(0.64-0.99)$ & .037 & $1.10(0.89-1.27)$ & .322 & - & - & - & - & - & - \\
\hline $\begin{array}{l}\text { Oral antihyperglycemic } \\
\text { drugs }\end{array}$ & $0.65(0.36-0.88)$ & .002 & $0.79(0.41-1.09)$ & .184 & - & - & - & - & - & - \\
\hline Diabetes on insulin & $1.15(1.02-1.27)$ & .028 & $1.01(0.67-1.27)$ & .921 & - & - & - & - & - & - \\
\hline Smoking & $1.10(1.01-1.19)$ & .034 & $1.02(0.86-1.16)$ & .745 & - & - & - & - & - & - \\
\hline Hypertension & $1.13(1.05-1.21)$ & .003 & $1.19(1.03-1.31)$ & .019 & - & - & - & - & - & - \\
\hline Poor mobility & $1.62(1.24-2.11)$ & $<.001$ & $0.66(0.51-1.28)$ & .354 & - & - & - & - & - & - \\
\hline Pulmonary hypertension & $2.73(2.50-2.98)$ & $<.001$ & $2.51(2.14-2.94)$ & $<.001$ & $1.29(1.24-1.35)$ & $<.001$ & $2.90(2.47-3.40)$ & $<.001$ & .365 & $<.001$ \\
\hline Chronic kidney disease & $0.94(0.82-1.08)$ & .379 & $1.17(0.94-1.35)$ & .145 & - & - & - & - & - & - \\
\hline Vascular disease & $1.41(1.31-1.50)$ & $<.001$ & $1.40(1.20-1.56)$ & $<.001$ & - & - & - & - & - & - \\
\hline Chronic lung disease & $1.29(1.19-1.38)$ & $<.001$ & $1.28(1.08-1.44)$ & .009 & $1.15(1.08-1.21)$ & $<.001$ & $1.30(1.16-1.42)$ & $<.001$ & .903 & .051 \\
\hline \multicolumn{11}{|l|}{$\operatorname{LVEF}(\%)$} \\
\hline$<20$ & $0.77(0.28-1.12)$ & .233 & $0.96(0.20-1.40)$ & .896 & - & - & - & - & - & - \\
\hline $20-30$ & $0.89(0.67-1.07)$ & .229 & $0.98(0.62-1.24)$ & .877 & - & - & - & - & - & - \\
\hline $30-50$ & $0.95(0.85-1.04)$ & .278 & $1.04(0.88-1.18)$ & .578 & - & - & - & - & - & - \\
\hline$>50$ & $1.05(0.96-1.12)$ & .264 & $0.97(0.80-1.11)$ & .677 & - & - & - & - & - & - \\
\hline CAD & & & & & - & - & - & - & - & - \\
\hline No CAD & $1.09(0.94-1.23)$ & .224 & $1.04(0.83-1.21)$ & .710 & - & - & - & - & - & - \\
\hline $1 \mathrm{VD}$ & $1.03(0.82-1.20)$ & .775 & $1.07(0.78-1.29)$ & .601 & - & - & - & - & - & - \\
\hline $2 \mathrm{VD}$ & $1.03(0.82-1.21)$ & .760 & $1.07(0.69-1.34)$ & .676 & - & - & - & - & - & - \\
\hline $3 \mathrm{VD}$ & $0.99(0.86-1.15)$ & .934 & $0.65(0.06-1.07)$ & .111 & - & - & - & - & - & - \\
\hline Previous MI & $1.06(0.94-1.20)$ & .314 & $1.06(0.94-1.20)$ & .089 & - & - & - & - & - & - \\
\hline Previous PCI & $1.22(1.10-1.33)$ & .001 & $1.34(1.14-1.50)$ & .003 & - & - & $1.22(1.01-1.40)$ & .045 & - & .472 \\
\hline \multicolumn{11}{|l|}{$\mathrm{CCS}$} \\
\hline No signs of angina & $0.56(0.45-0.71)$ & $<.001$ & $0.38(0.25-0.58)$ & $<.001$ & $0.84(0.80-0.89)$ & $<.001$ & - & - & .001 & - \\
\hline 1 & $0.51(0.41-0.64)$ & $<.001$ & $0.68(0.56-0.82)$ & $<.001$ & - & - & - & - & - & - \\
\hline 2 & $0.45(0.36-0.56)$ & $<.001$ & $1.88(1.20-2.95)$ & .006 & - & - & $1.23(1.07-1.37)$ & .006 & - & .078 \\
\hline 3 & $0.32(0.25-0.40)$ & $<.001$ & $2.00(1.38-2.58)$ & $<.001$ & - & - & $1.50(1.36-1.60)$ & $<.001$ & - & .185 \\
\hline 4 & $1.62(1.53-1.69)$ & $<.001$ & $2.45(1.54-3.91)$ & $<.001$ & - & - & $1.59(1.36-1.74)$ & $<.001$ & - & .082 \\
\hline \multicolumn{11}{|l|}{ NYHA } \\
\hline No signs of heart failure & $0.76(0.33-1.08)$ & .163 & $0.63(0.29-1.43)$ & .488 & - & - & - & - & - & - \\
\hline I & $0.77(0.46-1.02)$ & .078 & $1.14(0.81-1.38)$ & .359 & - & - & - & - & - & - \\
\hline II & $0.77(0.55-0.95)$ & .011 & $0.84(0.63-1.02)$ & .083 & - & - & - & - & - & - \\
\hline
\end{tabular}




\begin{tabular}{|c|c|c|c|c|c|c|c|c|c|c|}
\hline \multirow[b]{3}{*}{ Model } & \multicolumn{4}{|c|}{ Univariable } & \multicolumn{4}{|c|}{ Multivariable } & \multicolumn{2}{|c|}{$P$ for interaction } \\
\hline & \multicolumn{2}{|c|}{ Unadjusted hazard ratios } & \multicolumn{2}{|c|}{ After PS matching hazard ratios } & \multicolumn{2}{|c|}{ Unadjusted hazard ratios } & \multicolumn{2}{|c|}{ After PS matching hazard ratios } & \multirow{2}{*}{$\begin{array}{c} \\
\text { Unadjusted } P \\
\text { interaction }\end{array}$} & \multirow{2}{*}{$\begin{array}{c}\text { After PS } \\
\text { matching } P \text { for } \\
\text { interaction }\end{array}$} \\
\hline & HR $(95 \%$ CI $)$ & $P$ value & HR $(95 \%$ CI) & $P$ value & HR $(95 \%$ CI $)$ & $P$ value & HR $(95 \%$ CI $)$ & $P$ value & & \\
\hline III & $0.95(0.78-1.10)$ & .548 & $1.17(1.04-1.29)$ & .014 & - & - & $1.20(1.07-1.32)$ & .003 & - & .772 \\
\hline IV & $1.08(0.94-1.24)$ & 292 & $0.74(0.36-1.03)$ & .085 & - & - & - & - & - & - \\
\hline \multicolumn{11}{|l|}{ Procedural characteristics } \\
\hline Redo surgery & $1.31(1.21-1.40)$ & $<.001$ & $1.35(1.13-1.52)$ & .005 & $1.10(1.02-1.19)$ & .014 & $1.29(1.06-1.46)$ & 018 & .770 & 139 \\
\hline Endocarditis & $1.08(0.84-1.39)$ & .526 & $0.92(0.39-1.28)$ & .716 & - & - & - & - & - & - \\
\hline Cardiogenic shock & $1.13(0.69-1.87)$ & .620 & $1.02(0.22-1.46)$ & 944 & - & - & - & - & - & - \\
\hline Critical preoperative state & $0.83(0.31-1.20)$ & .417 & $0.95(0.30-1.35)$ & .841 & - & - & - & - & - & - \\
\hline IABP & $1.31(0.93-1.56)$ & .095 & $1.37(0.82-1.66)$ & 148 & - & - & $1.50(1.07-1.74)$ & .030 & - & .514 \\
\hline Intravenous inotropes & $1.09(0.74-1.34)$ & .574 & $0.93(0.40-1.29)$ & .757 & - & - & $1.35(1.11-1.52)$ & .006 & - & .736 \\
\hline Intravenous nitrates & $1.20(1.08-1.31)$ & .003 & $0.94(0.58-1.21)$ & 693 & - & - & - & - & - & - \\
\hline \multicolumn{11}{|l|}{ Mitral pathology } \\
\hline \multicolumn{11}{|l|}{ Mitral regurgitation grade } \\
\hline No regurgitation & $0.88(0.72-1.08)$ & .227 & $1.04(0.62-1.32)$ & .842 & - & - & - & - & - & - \\
\hline Trivial & $0.97(0.80-1.17)$ & .752 & $1.13(0.81-1.37)$ & .388 & - & - & - & - & - & - \\
\hline Mild & $0.92(0.79-1.06)$ & .249 & $1.13(0.88-1.32)$ & .276 & - & - & - & - & - & - \\
\hline Moderate & $0.97(0.88-1.06)$ & .493 & $1.11(1.07-1.39)$ & .04 & - & - & $1.23(1.00-1.40)$ & .046 & - & 299 \\
\hline Severe & $0.97(0.90-1.06)$ & .525 & $1.06(0.90-1.19)$ & .451 & - & - & - & - & - & - \\
\hline \multicolumn{11}{|l|}{ Mitral regurgitation } \\
\hline Primary & $0.90(0.79-1.00)$ & .062 & $0.94(0.75-1.11)$ & .512 & - & - & $0.78(0.55-0.97)$ & .021 & - & .368 \\
\hline Secondary & $1.10(1.00-1.21)$ & .062 & $1.06(0.89-1.25)$ & .512 & - & - & - & - & - & - \\
\hline Mitral valve stenosis & $1.19(0.84-1.44)$ & .674 & $1.03(0.50-1.39)$ & .857 & - & - & - & - & - & - \\
\hline \multicolumn{11}{|l|}{ Surgery } \\
\hline MV repair & $0.92(0.84-1.01)$ & .072 & $1.01(0.84-1.15)$ & 911 & $0.94(0.90-0.98)$ & .004 & - & - & .675 & - \\
\hline MV replacement & $1.08(0.99-1.16)$ & .072 & $0.99(0.85-1.16)$ & 911 & - & - & - & - & - & - \\
\hline Concomitant procedure & & & & & - & - & - & - & - & - \\
\hline $\mathrm{MV}+\mathrm{CABG}$ & $1.26(1.18-1.33)$ & $<.001$ & $2.48(1.56-3.94)$ & $<.001$ & - & - & - & - & - & - \\
\hline $\mathrm{MV}+\mathrm{AV}$ & $1.18(1.05-1.32)$ & .004 & $0.92(0.10-1.39)$ & .803 & - & - & - & - & - & - \\
\hline $\mathrm{MV}+\mathrm{TV}$ & $1.08(0.99-1.17)$ & .091 & $1.02(0.87-1.18)$ & .843 & $1.04(1.00-1.08)$ & .039 & - & - & .514 & - \\
\hline \multicolumn{11}{|l|}{ Status } \\
\hline Elective & $0.67(0.43-1.04)$ & .072 & $0.98(0.79-1.14)$ & .797 & - & - & - & - & - & - \\
\hline Urgent & $0.82(0.53-1.28)$ & .384 & $1.05(0.88-1.20)$ & .517 & $1.05(1.00-1.11)$ & .042 & - & - & .271 & - \\
\hline Emergency & $1.16(0.77-1.75)$ & .466 & $1.36(1.26-1.42)$ & 191 & - & - & $2.30(1.08-4.87)$ & .031 & - & 172 \\
\hline Late survival & $0.71(0.63-0.79)$ & $<.001$ & $0.82(0.70-0.96)$ & .011 & $0.92(0.87-0.96)$ & $<.001$ & $0.72(0.51-0.90)$ & .002 & $<.001$ & . 484 \\
\hline
\end{tabular}

$P S$, Propensity score; HR, hazard ratio; $C I$, confidence interval; EuroSCORE, European system for cardiac operative risk evaluation; $L V E F$, left ventricle ejection fraction; $C A D$, coronary artery disease; $V D$, vessel disease; $M I$, myocardial infarction; $P C I$, percutaneous coronary intervention; $C C S$, Canadian Cardiovascular Society; $N Y H A$, New York Heart Association; IABP, intra-aortic balloon pump; $M V$, mitral valve; $C A B G$, coronary artery bypass grafting; $A V$, aortic valve; $T V$, tricuspid valve. 